\title{
DIVERGENCE OPERATORS AND ODD POISSON BRACKETS
}

\author{
YVETTE KOSMANN-SCHWARZBACH AND JUAN MONTERDE
}

\begin{abstract}
We define the divergence operators on a graded algebra, and we show that, given an odd Poisson bracket on the algebra, the operator that maps an element to the divergence of the hamiltonian derivation that it defines is a generator of the bracket. This is the "odd laplacian", $\Delta$, of Batalin-Vilkovisky quantization. We then study the generators of odd Poisson brackets on supermanifolds, where divergences of graded vector fields can be defined either in terms of berezinian volumes or of graded connections. Examples include generators of the Schouten bracket of multivectors on a manifold (the supermanifold being the cotangent bundle where the coordinates in the fibres are odd) and generators of the KoszulSchouten bracket of forms on a Poisson manifold (the supermanifold being the tangent bundle, with odd coordinates on the fibres).
\end{abstract}

\section{INTRODUCTION}

Graded algebras with an odd Poisson bracket - also called Gerstenhaber algebras play an important role in the theory of deformations of algebraic structures as well as in several areas of field theory, as has been shown by Batalin and Vilkovisky [2] [3], Witten [43], Lian and Zuckerman [29], Getzler [10], Hata and Zwiebach [11], among others. Generators of odd Poisson brackets, in the sense of Equation (11) below, are differential operators of order 2 of the underlying graded algebra, sometimes called "odd laplacians", and usually denoted by the letter $\Delta$. Batalin-Vilkovisky algebras - BV-algebras, for short - are a special class of these algebras, those for whose bracket there exists a generator assumed to be of square 0 . The geometrical approach to odd Poisson algebras and BV-algebras in terms of supermanifolds was first developed by Leites [28], Khudaverdian [17] (see also [19]) and Schwarz [37].

The purpose of this article is to study various constructions of generators of odd Poisson brackets. Our constructions will rely on the general notion of divergence operator on a graded algebra, which generalizes the concept of the divergence of a vector field in elementary analysis. Given an odd Poisson bracket, to each element in the algebra we associate the divergence of the hamiltonian derivation that it defines, and we show that such a map from the algebra to itself, multiplied by the factor $\frac{1}{2}$ and by an appropriate sign, is a generator of that bracket (Theorem 1.2). We then adopt the language of supermanifolds to treat two constructions which determine divergence operators on the structural sheaf of the supermanifold.

The first construction uses berezinian volumes, and is modeled after the construction of divergence operators on smooth, purely even manifolds which uses volume elements. Once a divergence operator is defined, we apply Theorem 1.2 to obtain generators of an odd Poisson bracket on the supermanifold. One can deform any generator, obtained from a berezinian volume, by a change of berezinian volume, i.e., the multiplication by an invertible, even function. The deformed generator 
then differs from the original one by the addition of a hamiltonian derivation. If the original generator is of square 0 , a sufficient condition for a deformed generator to remain of square 0 is given by a Maurer-Cartan equation. (Under the name "BV quantum master equation", this condition plays a fundamental role in the BV quantization of gauge fields.) We study two special cases in detail: (i) the cotangent bundle of a smooth manifold viewed as a supermanifold whose structural sheaf is the sheaf of multivectors on the manifold, in which case the odd Poisson bracket under consideration is the Schouten bracket, and (ii) the tangent bundle of a smooth manifold as a supermanifold whose structural sheaf is the sheaf of differential forms on the manifold, when the underlying smooth, even manifold is a Poisson manifold.

The second construction utilizes graded linear connections on supermanifolds, and generalizes to the graded case an approach to the construction of divergence operators on purely even manifolds using linear connections. Given a linear connection on a smooth, even manifold, the divergence of a vector field $X$ is defined as the trace of the difference of the covariant derivation in the direction of $X$ and of the map $[X,$.$] ,$ where [, ] is the Lie bracket of vector fields. In the case of a supermanifold, given a graded linear connection, the divergence of a graded vector field is defined in a similar manner, replacing the trace by the supertrace, and the Lie bracket by the graded commutator. We then apply Theorem 1.2 to obtain a generator of an odd Poisson bracket on the supermanifold. As an example, we again study the generators of the Schouten bracket of multivectors on a manifold, equipped with a linear connection. On the one hand, there exists a unique generator of the Schouten bracket whose restriction to the vector fields is the divergence defined by the linear connection. On the other hand, on the cotangent bundle considered as a supermanifold, the linear connection on the manifold defines a graded metric in a simple way. This graded metric in turn determines a graded torsionless connection on this supermanifold the associated Levi-Civita connection -, from which we obtain a generator of the Schouten bracket, following our general procedure. We show that these two generators of the Schouten bracket coincide and that, when the connection is flat, this generator is of square 0 .

One can ask: what happens if we deal with an even Poisson bracket instead of an odd one? The answer is that the phenomena in the odd and in the even cases are very different, although there is a formal similarity of the constructions. In the even case, the results extend those of the purely even case, e.g., the usual case of Poisson algebras of smooth manifolds. Taking the divergence of a hamiltonian vector field with respect to a volume form if the manifold is orientable, or, more generally, to a density, yields a derivation of the algebra, i.e., a vector field. This is the modular vector field that has been studied in Poisson geometry (see [24], 42]) and in more general contexts (see [16], 44], 21]). One can prove that this vector field is closed in the Poisson-Lichnerowicz cohomology. A change in the volume element modifies the vector field by a hamiltonian vector field, therefore the cohomology class of the closed vector field does not change. One thus obtains a cohomology class, called "the modular class" [42]. So, while in the odd case we get a secondorder differential operator which is a generator of the bracket, in the even case we get a first-order differential operator, in fact a derivation of the structural sheaf of associative algebras. 
The paper is organized as follows. In Section 1, we first recall the definition of an odd Poisson bracket on a $\mathbb{Z}_{2}$-graded algebra, and, in Section 1 .2.2, we define algebraically the notion of a divergence operator on a graded algebra and that of its curvature. We then prove Theorem 1.2, which will serve as the main tool in our constructions, and we study the deformation of divergence operators and of the associated generators of odd Poisson brackets.

In Section 2, we study the divergence operators defined by berezinian volumes (Proposition 2.2), the associated generators of odd Poisson brackets, and their deformation under a change of berezinian volume. We show that the "Batalin-Vilkovisky quantum master equation" appears as a sufficient condition for the modified generator to remain of square 0 (Proposition 2.5). In Section 2.3, we consider the example of the cotangent bundle of a manifold $M$, considered as a supermanifold. To a volume element element $\mu$ on $M$, there corresponds a berezinian volume, which behaves like the "square of $\mu$ ". In Theorem 2.8, we show that the generator of the Schouten bracket furnished by the general construction outlined above coincides with the generator obtained from the de Rham differential by the isomorphism defined by $\mu$ relating forms to multivectors. In Section 2.4, we treat the case of the tangent bundle of $M$, which is an odd Poisson supermanifold when $M$ has a Poisson structure. We prove that the generator defined by the divergence of hamiltonian vector fields with respect to the canonical berezinian volume coincides with the Poisson homology operator, and that its square therefore vanishes (Theorem 2.11). In Section 2.5, we express the properties of the supermanifolds studied in Sections 2.3 and 2.4 in the language of QS, SP and QSP manifolds of [37] and [1] (Theorems 2.16 and 2.17).

In Section 3, we study the divergence operators defined by graded linear connections. The definitions of a graded linear connection, its curvature and torsion, and of the divergence operator that it defines (Proposition 3.3) are given in Section 3.1. We then study the generator associated to a torsionless graded linear connection, of an odd Poisson bracket and the effect of a change of connection on the generator (Section 3.2). The Levi-Civita connection of a graded metric on a supermanifold is introduced in Section 3.3. In the remaining part of Section 3, we study the cotangent bundle of a manifold as an odd Poisson supermanifold. More specifically we study two constructions of generators of the Schouten bracket associated to a torsionless linear connection on the base manifolds, and we show that the two constructions yield the same generator (Theorem 3.16). We conclude the paper with remarks concerning the relationships between divergence operators, right and left module structures in the theory of Lie-Rinehart algebras [15] and right and left $\mathcal{D}$-modules, and their analogues in the graded case [34, and we formulate a conjecture regarding the existence of a unique prolongation of a divergence operator on a supermanifold into a generator of an odd bracket on the algebra of graded multivectors.

We shall usually denote a supermanifold by a pair $(M, \mathcal{A})$ where $M$ is an ordinary smooth manifold, called the base manifold, and $\mathcal{A}$ is a sheaf over $M$ of $\mathbb{Z}_{2}$-graded commutative, associative algebras. The sections of $\mathcal{A}$ will be denoted by $f, g, \ldots$, but this notation will be modified in some instances. When $a$ is an element of a $\mathbb{Z}_{2}$-graded vector space, $|a|$ denotes the $\mathbb{Z}_{2}$-degree of $a$ and, whenever it appears in a formula, it is understood that $a$ is homogeneous. The word "graded" will often be omitted. The bracket [, ] denotes the graded commutator. Manifolds and maps 
are assumed to be smooth. We recall some general properties of supermanifolds and the definition of the berezinian volumes in the Appendix.

\section{Odd Poisson BRackets And Divergence operators}

In this section, we review the main definitions concerning odd Poisson brackets on graded algebras and we study how to construct generators of such brackets.

1.1. Gerstenhaber and $\mathbf{B V}$-algebras. Let $\mathbf{A}$ be a $\mathbb{Z}_{2}$-graded commutative, associative algebra over a field of characteristic 0 . The multiplication map of algebra $\mathbf{A}, \quad(f, g) \in \mathbf{A} \times \mathbf{A} \mapsto f g \in \mathbf{A}$, will be denoted by $m$. By definition, an odd Poisson bracket or a $\mathbb{Z}_{2}$-Gerstenhaber bracket on $\mathbf{A}$ is an odd bilinear map, $\pi:(f, g) \in \mathbf{A} \times \mathbf{A} \mapsto \llbracket f, g \rrbracket \in \mathbf{A}$, satisfying, for any $f, g, h \in \mathbf{A}$,

- $\llbracket f, g \rrbracket=-(-1)^{(|f|-1)(|g|-1)} \llbracket g, f \rrbracket \quad$ (skew-symmetry),

- $\llbracket f, \llbracket g, h \rrbracket \rrbracket=\llbracket \llbracket f, g \rrbracket, h \rrbracket+(-1)^{(|f|-1)(|g|-1)} \llbracket g, \llbracket f, h \rrbracket \rrbracket \quad$ (graded Jacobi identity),

$$
\text { - } \llbracket f, g h \rrbracket=\llbracket f, g \rrbracket h+(-1)^{(|f|-1)|g|} g \llbracket f, h \rrbracket \quad \text { (Leibniz rule) . }
$$

("Map $\pi$ is odd" means that $|\llbracket f, g \rrbracket|=|f|+|g|-1$ modulo 2.) The pair (A, $\pi$ ) is then called an odd Poisson algebra or a $\mathbb{Z}_{2}$-Gerstenhaber algebra.

A linear map of odd degree, $\Delta: \mathbf{A} \rightarrow \mathbf{A}$, such that, for all $f, g \in \mathbf{A}$,

$$
\llbracket f, g \rrbracket=(-1)^{|f|}\left(\Delta(f g)-(\Delta f) g-(-1)^{|f|} f(\Delta g)\right),
$$

is called a generator or a generating operator of $\pi$ (or of bracket 【, 『). If there exists a generator $\Delta$ of the bracket which is of square 0 , then $(\mathbf{A}, \pi, \Delta)$ is called a $\mathbb{Z}_{2}$-Batalin-Vilkovisky algebra, or $B V$-algebra for short.

Remark. Since a Gerstenhaber bracket in the usual sense, defined on a $\mathbb{Z}$-graded algebra, is of $\mathbb{Z}$-degree -1 , it is clear that it can also be considered to be a $\mathbb{Z}_{2^{-}}$ Gerstenhaber bracket. Similarly, Batalin-Vilkovisky algebras in the usual, $\mathbb{Z}$-graded sense, are particular cases of $\mathbb{Z}_{2}$-Batalin-Vilkovisky algebras.

A generator of an odd Poisson bracket is clearly not a derivation of the graded associative algebra $(\mathbf{A}, m)$, unless the bracket is identically 0 . We shall now see under what condition a generator $\Delta$ of an odd Poisson bracket $\pi$ is a derivation of the graded Lie algebra $(\mathbf{A}, \pi)$. A straightforward computation, using the defining relation (11) for a generator, yields the identity,

(2) $\Delta^{2}(f g)-\left(\Delta^{2} f\right) g-f\left(\Delta^{2} g\right)=(-1)^{|f|}\left(\Delta(\llbracket f, g \rrbracket)-\llbracket \Delta f, g \rrbracket-(-1)^{|f|-1} \llbracket f, \Delta g \rrbracket\right)$,

for all $f, g \in \mathbf{A}$. From (2), we obtain

Lemma 1.1. A generator $\Delta$ of an odd Poisson bracket $\pi$ is an odd derivation of the odd Poisson algebra, $(\mathbf{A}, \pi)$, if and only if the map $\Delta^{2}$ is an even derivation of the graded associative algebra $(\mathbf{A}, m)$. In particular, if $\Delta^{2}=0$, then $\Delta$ is a derivation of $(\mathbf{A}, \pi)$.

Remark. In the case of a $\mathbb{Z}$-graded algebra and a the linear map $\Delta$ of degree -1 , the map $\Delta^{2}$, which is of degree -2 , is a derivation of $(\mathbf{A}, m)$ if and only if it vanishes. Therefore, in this case, a generator $\Delta$ of $\pi$ is a derivation of $(\mathbf{A}, \pi)$ if and only if $\Delta^{2}=0$. 
The following identity, of which we shall make use in Section 2.5, is the result of another computation. If $D$ is an odd derivation of $(\mathbf{A}, m)$, then

$[D, \Delta](f g)-([D, \Delta] f) g-f([D, \Delta] g)=(-1)^{|f|}\left(D \llbracket f, g \rrbracket-\llbracket D f, g \rrbracket-(-1)^{|f|-1} \llbracket f, D g \rrbracket\right)$.

Let $\delta_{m}$ be the graded Hochschild differential of algebra $(\mathbf{A}, m)$. Equation (四) expresses the equality

$$
\pi(f, g)=(-1)^{|f|-1}\left(\delta_{m} \Delta\right)(f, g) .
$$

Let us also introduce the graded Chevalley-Eilenberg differential of $(\mathbf{A}, \pi)$, denoted by $\delta_{\pi}$. Equation (2) expresses the equality

$$
\left(\delta_{\pi} \Delta\right)(f, g)=(-1)^{|f|-1}\left(\delta_{m}\left(\Delta^{2}\right)\right)(f, g),
$$

and Lemma 1.1 can be reformulated as follows : $\Delta$ is a $\delta_{\pi}$-cocycle if and only if $\Delta^{2}$ is a $\delta_{m}$-cocycle.

1.2. Divergence operators. Let $(\mathbf{A}, m)$ be a $\mathbb{Z}_{2}$-graded commutative, associative algebra, and let Der $\mathbf{A}$ be the graded vector space of graded derivations of $(\mathbf{A}, m)$. By definition, a divergence operator on $\mathbf{A}$ is an even linear map, $\operatorname{div}: \operatorname{Der} \mathbf{A} \rightarrow \mathbf{A}$, such that

$$
\operatorname{div}(f D)=f \operatorname{div}(D)+(-1)^{|f||D|} D(f),
$$

for any $D \in$ Der $\mathbf{A}$ and any $f \in \mathbf{A}$.

This definition obviously generalizes the usual notion of divergence of vector fields in elementary analysis. More generally, if $\mathbf{A}$ is the purely even algebra of smooth real- or complex-valued functions on a smooth manifold, divergence operators on A can be defined by means of either volume forms or linear connections, the two approaches being related in a simple way. See [24]. These two approaches will be generalized below in Sections 2 and 3, respectively.

We define the curvature of a divergence operator, as the bilinear map, $\mathcal{R}^{\text {div }}$ : Der $\mathbf{A} \times \operatorname{Der} \mathbf{A} \rightarrow \mathbf{A}$ by

$$
\mathcal{R}^{\operatorname{div}}\left(D_{1}, D_{2}\right)=\operatorname{div}\left[D_{1}, D_{2}\right]-D_{1}\left(\operatorname{div} D_{2}\right)+(-1)^{\left|D_{1}\right|\left|D_{2}\right|} D_{2}\left(\operatorname{div} D_{1}\right) .
$$

for any derivations $D_{1}, D_{2} \in$ Der $\mathbf{A}$. A short computation shows that $\mathcal{R}^{\text {div }}$ is $\mathbf{A}$ bilinear. If $\delta_{[,,]}$denotes the graded Chevalley-Eilenberg differential of the graded Lie algebra (Der $\mathbf{A},[$,$] ) acting on cochains on Der \mathbf{A}$ with values in the Der $\mathbf{A}$-module A, then the definition of $\mathcal{R}^{\text {div }}$ in (5) can be written

$$
\mathcal{R}^{\operatorname{div}}=\delta_{[,,]}(\operatorname{div}) \text {. }
$$

1.3. Divergence operators and generators. On the odd Poisson algebra $(\mathbf{A}, \pi)$, we define the hamiltonian mapping by $X^{\pi}: \mathbf{A} \rightarrow$ Der $\mathbf{A}$, defined by

$$
f \mapsto X_{f}^{\pi}=\llbracket f, . \rrbracket .
$$

The graded Jacobi identity for the bracket $\pi$ is equivalent to the relation

$$
X_{\llbracket f, g \rrbracket}^{\pi}=\left[X_{f}^{\pi}, X_{g}^{\pi}\right],
$$

for any $f, g \in \mathbf{A}$, which expresses the fact that $X^{\pi}$ is a morphism of graded Lie algebras from $(\mathbf{A}, \pi)$ to (Der $\mathbf{A},[]$,$) .$ 
We now introduce the main object of interest in this paper, the odd linear map $\Delta: \mathbf{A} \rightarrow \mathbf{A}$, depending on both $\pi$ and the choice of a divergence operator, defined by

$$
\Delta f=(-1)^{|f|} \frac{1}{2} \operatorname{div}\left(X_{f}^{\pi}\right)
$$

for $f \in \mathbf{A}$.

Theorem 1.2. The operator $\Delta$ on $\mathbf{A}$, defined by $(\overline{0})$, is a generator of bracket $\pi$.

Proof. To show that Equation (11) is satisfied, we compute $\Delta(f g)$ using the Leibniz rule for the odd Poisson bracket, and the fundamental property (雨) of the divergence operators,

$$
\begin{aligned}
& (-1)^{|f|+|g|} \Delta(f g)=\frac{1}{2} \operatorname{div} X_{f g}^{\pi}=\frac{1}{2} \operatorname{div}\left(f X_{g}^{\pi}+(-1)^{|f||g|} g X_{f}^{\pi}\right) \\
& =(-1)^{|g|} f \Delta g+(-1)^{|f|(|g|+1)} g \Delta f+\frac{1}{2}(-1)^{|f|(|g|+1)} \llbracket g, f \rrbracket+\frac{1}{2}(-1)^{|g|} \llbracket f, g \rrbracket \\
& =(-1)^{|g|} f \Delta g+(-1)^{|f|+|g|}(\Delta f) g+(-1)^{|g|} \llbracket f, g \rrbracket,
\end{aligned}
$$

and the result follows.

This fundamental result must be contrasted with a parallel, but strikingly different result valid for even Poisson brackets, and consequently also, in the usual case, for ungraded Poisson algebras. If, in (7), we replace the hamiltonian operator defined by an odd Poisson bracket by the one defined by an even Poisson bracket, a computation similar to the proof of Theorem 1.2 shows that the operator thus defined is a derivation of the associative multiplication. When the Poisson algebra is the algebra of functions of a smooth orientable Poisson manifold, in which case the divergence operator is the one associated with a volume element, this derivation is a vector field. It is easy to see that, up to the factor $1 / 2$, it coincides with the modular vector field of the Poisson manifold [42] (also [16], 21] and references cited therein).

We now establish a relation between the operator $\Delta$ defined by $\pi$ and div, and the curvature of the divergence operator evaluated on hamiltonian derivations.

Proposition 1.3. For any $f, g \in \mathbf{A}$,

$$
\Delta \llbracket f, g \rrbracket-\llbracket \Delta f, g \rrbracket-(-1)^{|f|-1} \llbracket f, \Delta g \rrbracket=(-1)^{|f|+|g|-1} \frac{1}{2} \mathcal{R}^{\operatorname{div}}\left(X_{f}^{\pi}, X_{g}^{\pi}\right) .
$$

Proof. By (6) and the definition of $\Delta$,

$$
\begin{aligned}
& \Delta \llbracket f, g \rrbracket=(-1)^{|f|+|g|-1} \frac{1}{2} \operatorname{div} X_{\llbracket f, g \rrbracket}^{\pi}=(-1)^{|f|+|g|-1} \frac{1}{2} \operatorname{div}\left[X_{f}^{\pi}, X_{g}^{\pi}\right], \\
& \llbracket f, \Delta g \rrbracket=(-1)^{|g|} \frac{1}{2} X_{f}^{\pi}\left(\operatorname{div} X_{g}^{\pi}\right), \quad \llbracket \Delta f, g \rrbracket=(-1)^{|f||g|-1} \frac{1}{2} X_{g}^{\pi}\left(\operatorname{div} X_{f}^{\pi}\right) .
\end{aligned}
$$

In view of the definition of the curvature, the proposition follows.

Corollary 1.4. The generator $\Delta$ is a derivation of $(\mathbf{A}, \pi)$ if and only if $\mathcal{R}^{\text {div }}$ vanishes on the hamiltonian derivations. 
In terms of the differentials $\delta_{\pi}$ and $\delta_{[,]}$, Equation (8) can be written

$$
\left(\delta_{\pi} \Delta\right)(f, g)=(-1)^{|f|+|g|-1} \frac{1}{2}\left(\delta_{[,]} \operatorname{div}\right)\left(X_{f}^{\pi}, X_{g}^{\pi}\right) .
$$

1.4. Deformations of divergence operators and of generators. Since the difference of two divergence operators is an $\mathbf{A}$-linear map from Der $\mathbf{A}$ to $\mathbf{A}$, the space of divergence operators on $\mathbf{A}$ is an affine space over $\operatorname{Hom}_{\mathbf{A}}(\operatorname{Der} \mathbf{A}, \mathbf{A})$. We shall be interested in the case where the difference of two divergence operators is an evaluation map, $D \in$ Der $\mathbf{A} \mapsto D(2 w) \in \mathbf{A}$, where $w$ is a fixed, even element in $\mathbf{A}$. (The factor 2 is conventional.)

Since the difference of two generators of an odd Poisson bracket $\pi$ on $\mathbf{A}$ is a derivation of $(\mathbf{A}, m)$, the space of generators of $\pi$ is an affine space over Der $\mathbf{A}$.

Proposition 1.5. Let div and div' $^{\prime}$ be divergence operators on $\mathbf{A}$ such that there exists an even $w \in \mathbf{A}$ satisfying

$$
\operatorname{div}^{\prime} D=\operatorname{div} D+D(2 w)
$$

for all $D \in$ Der A. For $\pi$ a fixed odd Poisson bracket, let $\Delta$ and $\Delta^{\prime}$ be the generators of the bracket defined by (17) for div and $\operatorname{div}^{\prime}$, respectively. Then

$$
\Delta^{\prime}=\Delta+X_{w}^{\pi} .
$$

Proof. Equation (10) follows from (9) and the skew-symmetry of the bracket.

Remark. In the case of an even Poisson algebra, and, in particular, in the usual case of the Poisson algebra of a smooth manifold, a similar argument is valid. As a consequence, one proves that the class of the modular vector field in the Poisson cohomology is well-defined, independently of the choice of a volume element [42].

We shall now consider under what condition a generator of $\pi$ with vanishing square remains of square 0 when modified by the addition of an interior derivation $X_{w}^{\pi}$.

Proposition 1.6. If $\Delta$ is a generator of square 0 of bracket $\pi$, and $w$ is an even element of $\mathbf{A}$, then

$$
\left(\Delta+X_{w}^{\pi}\right)^{2}=X_{\Delta w+\frac{1}{2} \llbracket w, w \rrbracket}^{\pi} .
$$

Proof. From Lemma 1.1, we know that $\Delta^{2}=0$ implies that $\Delta$ is a derivation of $\pi$, whence $\left[\Delta, X_{w}^{\pi}\right]=X_{\Delta w}^{\pi}$. Using this relation and (6), we obtain

$$
\left(\Delta+X_{w}^{\pi}\right)^{2}=\left[\Delta, X_{w}^{\pi}\right]+\frac{1}{2}\left[X_{w}^{\pi}, X_{w}^{\pi}\right]=X_{\Delta w}^{\pi}+\frac{1}{2} X_{\llbracket w, w \rrbracket}^{\pi},
$$

whence the result.

The equation

$$
\Delta w+\frac{1}{2} \llbracket w, w \rrbracket=0,
$$

is the Maurer-Cartan equation, familiar from deformation theory. (See [39].) Thus we can state 
Corollary 1.7. Let $\Delta$ be a generator of square 0 of bracket $\pi$, and let $w$ be an even element of $\mathbf{A}$. The generator $\Delta^{\prime}=\Delta+X_{w}^{\pi}$ is of square 0 if and only if the hamiltonian operator $X_{\Delta w+\frac{1}{2} \llbracket w, w \rrbracket}^{\pi}$ vanishes. If, in particular, $w$ satisfies the MaurerCartan equation (12), then the generator $\Delta^{\prime}=\Delta+X_{w}^{\pi}$ is of square 0 .

\section{Berezinian volumes and generators of odd Poisson Brackets}

An odd Poisson supermanifold (resp., a $B V$-supermanifold) is a supermanifold $(M, \mathcal{A})$ whose sheaf of functions, $\mathcal{A}$, is a sheaf of odd Poisson algebras (resp., of BV-algebras). In the context of supermanifold theory, an odd Poisson bracket is often referred to as an antibracket [2] [43] [11] or a Buttin bracket 28]. The notions of derivations and divergence operators that we have introduced in Section 1 have obvious analogues in the case of sheaves of algebras, and we shall use the same symbols. On a supermanifold, a derivation of the sheaf of functions is called a graded vector field. We use the term "operator from $\mathcal{A}_{1}$ to $\mathcal{A}_{2}$ " for a morphism of sheaves of vector spaces from $\mathcal{A}_{1}$ to $\mathcal{A}_{2}$.

In this section, we show how generators of an odd Poisson bracket on a supermanifold can be obtained from berezinian volumes.

2.1. Divergence operators defined by berezinian volumes. We first recall the main properties of the Lie derivatives of berezinian sections. (See the Appendix for the definition of the berezinian sheaf. See, e.g., [7] for a proof of the following proposition.)

Proposition 2.1. Let $\xi$ be a berezinian section on $(M, \mathcal{A})$. For any graded vector field $D$ and any section $f$ of $\mathcal{A}$,

$$
\mathcal{L}_{D}(\xi . f)=\mathcal{L}_{D}(\xi) . f+(-1)^{|D \|| \xi \mid} \xi . D(f)
$$

and

$$
\mathcal{L}_{f . D}(\xi)=(-1)^{|f|(|D|+|\xi|)} \mathcal{L}_{D}(\xi . f) .
$$

We shall now define the divergence operator associated with a berezinian volume..

Proposition 2.2. Let $\xi$ be a berezinian volume. For any graded vector field $D$, there exists a unique section, $\operatorname{div}_{\xi}(D)$, of $\mathcal{A}$ such that

$$
\mathcal{L}_{D} \xi=(-1)^{|D||\xi|} \xi \cdot \operatorname{div}_{\xi}(D) .
$$

The map $D \mapsto \operatorname{div}_{\xi}(D)$ from $\operatorname{Der} \mathcal{A}$ to $\mathcal{A}$ defined by (15) is a divergence operator.

Proof. The map $\operatorname{div}_{\xi}$ is even, since $|\xi|+\left|\operatorname{div}_{\xi}(D)\right|=\left|\xi \cdot \operatorname{div}_{\xi}(D)\right|=\left|\mathcal{L}_{D} \xi\right|=|D|+|\xi|$. We must prove that $\operatorname{div}_{\xi}$ satisfies (14). Using Proposition 2.1, we obtain

$$
\begin{aligned}
\xi \cdot \operatorname{div}_{\xi}(f D) & =(-1)^{(|f|+|D|)|\xi|} \mathcal{L}_{f D} \xi=(-1)^{|D|(|f|+|\xi|)} \mathcal{L}_{D}(\xi . f) \\
& =(-1)^{|D|(|f|+|\xi|)}\left(\mathcal{L}_{D}(\xi) \cdot f+(-1)^{|D||\xi|} \xi . D(f)\right) \\
& =(-1)^{|f||D|}\left(\xi \cdot \operatorname{div}_{\xi}(D) f+\xi . D(f)\right)
\end{aligned}
$$

whence the result.

ExAmple. If $(M, \mathcal{A})=\mathbb{R}^{m \mid n}$ with graded coordinates $\left(x^{1}, \ldots, x^{m}, s^{1}, \ldots, s^{n}\right)$, the section

$$
\xi=\left[\mathrm{d}^{G} x^{1} \wedge \cdots \wedge \mathrm{d}^{G} x^{m} \otimes \frac{\partial}{\partial s^{1}} \circ \cdots \circ \frac{\partial}{\partial s^{n}}\right]
$$


is a berezinian volume and, if $D=\sum_{i=1}^{m} g^{i} \frac{\partial}{\partial x^{i}}+\sum_{\rho=1}^{n} h^{\rho} \frac{\partial}{\partial s^{\rho}}$, then

$$
\operatorname{div}_{\xi}(D)=\sum_{i=1}^{m} \frac{\partial g^{i}}{\partial x^{i}}+\sum_{\rho=1}^{n}(-1)^{\left|h^{\rho}\right|} \frac{\partial h^{\rho}}{\partial s^{\rho}}
$$

A short computation shows that, for graded vector fields $D_{1}$ and $D_{2}$,

$$
\operatorname{div}_{\xi}\left[D_{1}, D_{2}\right]=D_{1}\left(\operatorname{div}_{\xi} D_{2}\right)-(-1)^{\left|D_{1}\right|\left|D_{2}\right|} D_{2}\left(\operatorname{div}_{\xi} D_{1}\right) .
$$

In view of the definition of the curvature of a divergence operator (5), we have proved

Proposition 2.3. For any berezinian volume $\xi$, the curvature $\mathcal{R}^{\operatorname{div}_{\xi}}$ of the divergence operator $\operatorname{div}_{\xi}$ vanishes.

We now consider the effect on the divergence operator of a change of berezinian volume. When $v$ is an invertible, even section of $\mathcal{A}$, then $\xi . v$ is also a generator of the berezinian sheaf. We remark that any invertible, even section, $v$ of $\mathcal{A}$, can be written as $\pm e^{2 w}$ for an even section $w$ of $\mathcal{A}$. In fact, $v$ can be written as the product of a nowhere vanishing function on the base manifold and a function $1+u$, where $u$ is nilpotent. Since $u$ is nilpotent, say of order $k, 1+u$ is equal to $\exp (\ln (1+u))$, where $\ln (1+u)=\sum_{j=1}^{k}(-1)^{j-1} \frac{u^{j}}{j}$.

Proposition 2.4. Let $\xi$ be a berezinian volume. For any invertible, even section $v$ of $A$, the berezinian section $\xi . v$ is a berezinian volume, and, for any graded vector field $D$,

$$
\operatorname{div}_{\xi \cdot v}(D)=\operatorname{div}_{\xi}(D)+v^{-1} D(v) .
$$

If $v=e^{2 w}$, where $w$ is an even section of $\mathcal{A}$, then

$$
\operatorname{div}_{\xi \cdot e^{2 w}}(D)=\operatorname{div}_{\xi}(D)+D(2 w) .
$$

Proof. Using (13), we compute

$$
\begin{aligned}
(\xi . v) \cdot \operatorname{div}_{\xi . v}(D) & =(-1)^{|D||\xi|} \mathcal{L}_{D}(\xi . v) \\
& =(-1)^{|D||\xi|} \mathcal{L}_{D}(\xi) . v+\xi . D(v) \\
& =\xi \cdot\left(\operatorname{div}_{\xi}(D) v\right)+\xi . D(v) .
\end{aligned}
$$

Therefore

$$
v \operatorname{div}_{\xi . v}(D)=\operatorname{div}_{\xi}(D) v+D(v)
$$

and, multiplying both sides by $v^{-1}$, we obtain formula (17), since $v$ is even.

2.2. Properties of generators defined by berezinian volumes. We shall now assume that there is an odd Poisson structure, $\pi$, on $(M, \mathcal{A})$, with odd Poisson bracket $\llbracket, \rrbracket$. Let $\xi$ be a berezinian volume on $(M, \mathcal{A})$. Following the general pattern of Section 1.3, we define the operator $\Delta^{\pi, \xi}: \mathcal{A} \rightarrow \mathcal{A}$ by

$$
\Delta^{\pi, \xi} f=(-1)^{|f|} \frac{1}{2} \operatorname{div}_{\xi} X_{f}^{\pi},
$$

for any section $f$ of $\mathcal{A}$. It follows from Proposition 2.2 and Theorem 1.2 that the odd operator $\Delta^{\pi, \xi}$ is a generator of bracket $\pi$. Thus, given an odd Poisson bracket, to any berezinian volume there corresponds a generator of this bracket. We shall now 
study the effect on the generator of a change of berezinian volume, and determine under which conditions the generator corresponding to a berezinian volume is of square 0 .

It follows from $(18)$ and Proposition 1.5 that, when $\xi$ is a berezinian volume and $v=e^{2 w}$ an invertible, even section of $\mathcal{A}$,

$$
\Delta^{\pi, \xi \cdot v}=\Delta^{\pi, \xi}+X_{w}^{\pi} .
$$

It also follows from Proposition 1.6 that, if $\xi$ is a berezinian volume such that $\left(\Delta^{\pi, \xi}\right)^{2}=0$, and $v=e^{2 w}$ is an invertible, even section of $\mathcal{A}$, then,

$$
\left(\Delta^{\pi, \xi \cdot v}\right)^{2}=X_{\Delta^{\pi, \xi} w+\frac{1}{2} \llbracket w, w \rrbracket}^{\pi}
$$

Moreover,

$$
e^{-w} \Delta^{\pi, \xi}\left(e^{w}\right)=\frac{1}{2} e^{-w} \operatorname{div}_{\xi}\left(e^{w} X_{w}^{\pi}\right)=\Delta^{\pi, \xi} w+\frac{1}{2} e^{-w} \llbracket w, e^{w} \rrbracket,
$$

and therefore

$$
\Delta^{\pi, \xi} w+\frac{1}{2} \llbracket w, w \rrbracket=e^{-w} \Delta^{\pi, \xi} e^{w} .
$$

In the context of supermanifolds (usually infinite-dimensional), the Maurer-Cartan equation,

$$
\Delta^{\pi, \xi} w+\frac{1}{2} \llbracket w, w \rrbracket=0,
$$

is referred to as the Batalin-Vilkovisky quantum master equation. In the case of odd symplectic supermanifolds, the results stated below can be found in articles dealing with the BV-quantization of gauge theories, starting with [2], [3], followed by, among others, [43], [17], [37], [18], [1], or of string theories [11]. See also [10] and [39]. In our treatment, the more general case of possibly degenerate odd Poisson structures is included.

Proposition 2.5. Let $\xi$ be a berezinian volume on $(M, \mathcal{A})$ such that $\left(\Delta^{\pi, \xi}\right)^{2}=0$, and let $v=e^{2 w}$ be an invertible, even section of $\mathcal{A}$.

(i) The following conditions are equivalent

- $\Delta^{\pi, \xi}\left(e^{w}\right)=0$,

- $w$ is a solution of (23).

(ii) If this condition is satisfied, then $\left(\Delta^{\pi, \xi \cdot v}\right)^{2}=0$.

Proof. These implications follow from Equations (22) and (20).

Conversely $\left(\Delta^{\pi, \xi \cdot v}\right)^{2}=0$ implies that there exists an odd Casimir section $C$ of square 0 such that $\Delta^{\pi, \xi}\left(e^{w}\right) C=0$. (A Casimir section is a section of $\mathcal{A}$ such that $X_{C}^{\pi}=0$.) In fact, if $\left(\Delta^{\pi, \xi \cdot v}\right)^{2}=0$, then there exists a Casimir section $C$ such that $e^{-w} \Delta^{\pi, \xi}\left(e^{w}\right)=C$, or $\Delta^{\pi, \xi}\left(e^{w}\right)=e^{w} C$. Together with $\left(\Delta^{\pi, \xi}\right)^{2}=0$, this condition implies that $\Delta^{\pi, \xi}\left(e^{w}\right) C=0$, whence also $C^{2}=0$.

If the odd Poisson bracket $\pi$ is nondegenerate, any Casimir section is a constant, therefore even, and necessarily $C=0$. In this case, $(i)$ and $(i i)$ in the proposition are equivalent.

In quantum field theory, $e^{\frac{i}{\hbar} S}$ is the action, and the condition $\Delta^{\pi, \xi}\left(e^{\frac{i}{\hbar} S}\right)$ states that the action is closed with respect to the differential $\Delta^{\pi, \xi}$. 
Proposition 2.6. If $v=e^{2 w}$ is an invertible, even section of $\mathcal{A}$ such that $w$ is a solution of the Equation (23), then, for any section $f$ of $\mathcal{A}$,

$$
\Delta^{\pi, \xi \cdot v} f=e^{-w} \Delta^{\pi, \xi}\left(e^{w} f\right) .
$$

Proof. Using (1), we find that

$$
e^{-w} \Delta^{\pi, \xi}\left(e^{w} f\right)=e^{-w}\left(\llbracket e^{w}, f \rrbracket+\left(\Delta^{\pi, \xi} e^{w}\right) f+e^{w}\left(\Delta^{\pi, \xi} f\right)\right) .
$$

By (22) and (20),

$$
e^{-w} \Delta^{\pi, \xi}\left(e^{w} f\right)=\Delta^{\pi, \xi \cdot v}(f)+\left(\Delta^{\pi, \xi} w+\frac{1}{2} \llbracket w, w \rrbracket\right) f .
$$

The result is proved in view of $(20)$.

2.3. The supermanifold $\Pi T^{*} M$. For any manifold $M$ of dimension $n$, we consider the supermanifold $\Pi T^{*} M$ of dimension $n \mid n$, whose structural sheaf is the sheaf of multivectors on $M$. The supermanifold $\Pi T^{*} M$ has an odd Poisson bracket, the Schouten bracket of multivectors on $M$. It is in fact nondegenerate, i.e., the odd Poisson structure on $\Pi T^{*} M$ is symplectic. Here we revert to the usual notations for vector fields, differential forms and functions on the ordinary manifold $M$. See the Appendix for the definition to be used below of the map $\alpha \mapsto \tilde{\alpha}$ from forms on a supermanifold $(M, \mathcal{A})$ to forms on $M$.

Lemma 2.7. Given a volume form $\mu$ on $M$, there is a unique berezinian volume $\xi_{\mu}$ on $\Pi T^{*} M$ such that

$$
\widetilde{\xi_{\mu}(X)}=\left(i_{X_{(n)}} \mu\right) \mu
$$

for any field of multivectors $X$, where $i_{X_{(n)}} \mu$ is the result of the duality-pairing of the homogeneous component $X_{(n)}$ of degree $n$ of the multivector $X$ and the $n$-form $\mu$.

Proof. To any graded vector field, $D$, on $\Pi T^{*} M$ is associated a vector field, $\widetilde{D}$, on $M$, defined by

$$
\widetilde{D}(f)=\widetilde{D(f)}
$$

for any function $f$ on $M$. Given a differential $n$-form, $\mu$, on $M$, we can define a graded $n$-form, $\mu^{G}$, on $\Pi T^{*} M$ by

$$
<D_{1}, \ldots, D_{n}, \mu^{G}>=\mu\left(\widetilde{D_{1}}, \ldots, \widetilde{D_{n}}\right),
$$

for graded vector fields $D_{1}, \ldots, D_{n}$. Then $\widetilde{\mu^{G}}=\mu$. Since the map $X \mapsto i_{X_{(n)}} \mu$ is a differential operator of order $n$ on the structural sheaf of $\Pi T^{*} M$, the map $\xi_{\mu}: X \mapsto\left(i_{X_{(n)}} \mu\right) \mu^{G}$ defines a section of the berezinian sheaf, which is a berezinian volume if and only if $\mu$ is a volume form.

We remark that, for any positive function $v$ on $M$,

$$
\xi_{v \mu}=\left(\xi_{\mu}\right) \cdot v^{2}
$$

We assume that $M$ is an orientable manifold, and we let $\mu$ be a volume form on $M$. In the non orientable case, densities must be used instead of volume forms. On $\Pi T^{*} M$, there is an operator $\Delta^{\text {Schouten, } \xi_{\mu}}$ associated to the odd Poisson bracket and to the berezinian volume $\xi_{\mu}$ by means of (19). Let $\mathrm{d}$ be the de Rham differential on $M$, and let $*_{\mu}$ be the isomorphism from multivectors to forms defined by the volume 
form $\mu$. Then we know (see, e.g., 21]) that $\partial_{\mu}=-*_{\mu}^{-1} \mathrm{~d} *_{\mu}$ is a generator of the Schouten bracket.

Theorem 2.8. For any volume form $\mu$ on $M$, the generator $\Delta^{\text {Schouten, } \xi_{\mu}}$ of the Schouten bracket coincides with $\partial_{\mu}=-*_{\mu}^{-1} \mathrm{~d} *_{\mu}$, and $\left(\Delta^{\text {Schouten }, \xi_{\mu}}\right)^{2}=0$.

Proof. It is enough to show that these operators coincide on vector fields. We prove this fact using local coordinates $\left(x^{1}, \ldots, x^{n}, \xi_{1}, \ldots, \xi_{n}\right)$ on $\Pi T^{*} M$. For a vector field $X=\sum_{i=1}^{n} X^{i} \frac{\partial}{\partial x^{i}}$ considered as a function $\sum_{i=1}^{n} X^{i} \xi_{i}$ on $\Pi T^{*} M$,

$$
\llbracket X, . \rrbracket=\sum_{i=1}^{n} X^{i} \frac{\partial}{\partial x^{i}}-\sum_{i=1}^{n} \sum_{j=1}^{n} \frac{\partial X^{i}}{\partial x^{j}} \xi_{i} \frac{\partial}{\partial \xi_{j}} .
$$

Assume that $\mu_{0}=d x^{1} \wedge \ldots \wedge d x^{n}$, so that

$$
-\frac{1}{2} \operatorname{div}_{\xi_{\mu_{0}}} \llbracket X, . \rrbracket=-\frac{1}{2} \sum_{i=1}^{n}\left(\frac{\partial X^{i}}{\partial x^{i}}+\frac{\partial X^{i}}{\partial x^{i}}\right)=-\operatorname{div}_{\mu_{0}} X=\partial_{\mu_{0}} X .
$$

More generally, if $\mu=e^{w} \mu_{0}$, then $\xi_{\mu}=\xi_{\mu_{0}} \cdot e^{2 w}$, and for any vector field $X$,

$$
-\frac{1}{2} \operatorname{div}_{\xi_{\mu}} \llbracket X, . \rrbracket=-\frac{1}{2} \operatorname{div}_{\mu_{0}} X-X . w=\partial_{\mu_{0}} X-X . w=\partial_{e^{w} \mu_{0}} X=\partial_{\mu} X .
$$

The fact that $\left(\Delta^{\text {Schouten }, \xi_{\mu}}\right)^{2}=0$ is now an immediate consequence of the fact that $\mathrm{d}^{2}=0$.

Remark. The equality $\Delta=\partial_{\mu}$ means that, for any differential form $\alpha$,

$$
*_{\mu}^{-1} d \alpha=-\Delta *_{\mu}^{-1} \alpha
$$

The map $*_{\mu}^{-1}$ coincides with the "Fourier transform with respect to the odd variables" introduced in 41] and [37], p. 255. Therefore, in the case of a nondegenerate Poisson structure, our result reduces to that of [41] and [37], formula (20), a fact already observed by Witten in [43], formula (13). In the terminology of Voronov and Schwarz, the operator $\partial_{\mu}$ on the functions on $\Pi T^{*} M$ is the "Fourier transform" of the de Rham differential acting on functions on ПTM. Schwarz proves that a supermanifold $(M, \mathcal{A})$ of dimension $n \mid n$ with an odd symplectic structure is equivalent in a suitable sense to $\Pi T^{*} M$ with its canonical, odd symplectic structure.

Now, let $\mu$ be a volume form, and $\omega$ a differential form on $M$ such that $\omega_{(n)}$ is a volume form. If $Q=*_{\mu}^{-1} \omega$, then $\xi^{\prime}=\xi_{\mu} \cdot Q^{2}$ is a berezinian volume on $\Pi T^{*} M$. Setting $\Delta^{\prime}=\Delta^{\text {Schouten, } \xi^{\prime}}$, we see from Proposition 2.5 that $\left(\Delta^{\prime}\right)^{2}=0$ if $\Delta Q=0$. By Theorem 2.8, this condition is equivalent to $\mathrm{d} \omega=0$. So $\left(\Delta^{\prime}\right)^{2}=0$ if $\omega$ is a closed form. This result constitutes part of Theorem 5 of [37]. Moreover, it is proved there that two closed forms in the same de Rham cohomology class yield equivalent structures. 
2.4. The supermanifold $\Pi T M$. We shall now consider another supermanifold attached to a smooth manifold $M$ of dimension $n$. Let $\mathcal{A}=\Omega(M)$ be the sheaf of differential forms on $M$. The pair $(M, \mathcal{A})$, usually denoted by $\Pi T M$, is a supermanifold of dimension $n \mid n$. The sections of the sheaf $\Omega(M)$ will be denoted by $\alpha, \beta \ldots$, but differential forms of $\mathbb{Z}$-degree 0 , i.e., smooth functions, will be denoted by, $f, g, \ldots$ If $\alpha$ is a section of $\Omega(M)$, then we denote the homogeneous component of $\alpha$ of degree $n$ by $\alpha_{(n)}$.

\subsubsection{Canonical berezinian volume on the supermanifold $\Pi T M$.}

Lemma 2.9. There is a unique berezinian volume $\xi$ on $\Pi T M$, such that, for any section $\alpha$ of $\Omega(M)$,

$$
\widetilde{\xi(\alpha)}=\alpha_{(n)}
$$

Proof. If $\xi$ and $\xi^{\prime}$ are berezinian volumes satisfying $(\overline{24})$, then $\left(\overline{\left.\xi-\xi^{\prime}\right)(\alpha)}=0\right.$ for any section $\alpha$ of $\Omega(M)$, and this means, by the definition of the berezinian sheaf, that $\xi=\xi^{\prime}$, which proves the uniqueness of a berezinian volume satisfying (24). To prove its existence, we use local coordinates and we show the invariance under a change of coordinates. Let $\left(x^{1}, \ldots, x^{n}\right)$ be local coordinates on an open set $U$ of the manifold $M$. Then $\left(x^{1}, \ldots, x^{n}, s^{1}=\mathrm{d} x^{1}, \ldots, s^{n}=\mathrm{d} x^{n}\right)$ are graded local coordinates in $\Pi T M$, and a local basis of derivations is

$$
\left(\mathcal{L}_{\frac{\partial}{\partial x^{1}}}, \ldots, \mathcal{L}_{\frac{\partial}{\partial x^{n}}}, \frac{\partial}{\partial s^{1}}=i_{\frac{\partial}{\partial x^{1}}}, \ldots, \frac{\partial}{\partial s^{n}}=i_{\frac{\partial}{\partial x^{n}}}\right) .
$$

We now consider the local section of the berezinian sheaf,

$$
\xi_{U}=\left[\mathrm{d}^{G} x^{1} \wedge \cdots \wedge \mathrm{d}^{G} x^{n} \otimes i_{\frac{\partial}{\partial x^{1}} \wedge \cdots \wedge \frac{\partial}{\partial x^{n}}}\right] .
$$

A change of coordinates from $\left(x^{1}, \ldots, x^{n}\right)$ to $\left(y^{1}, \ldots, y^{n}\right)$ induces a change of graded coordinates to $\left(y^{1}, \ldots, y^{n}, t^{1}=\mathrm{d} y^{1}, \ldots, t^{n}=\mathrm{d} y^{n}\right)$, with matrix

$$
\left(\begin{array}{cc}
\left(\frac{\partial y^{i}}{\partial x^{k}}\right) & 0 \\
0 & \left(\frac{\partial y^{i}}{\partial x^{k}}\right)
\end{array}\right)
$$

whose berezinian is equal to 1 . Therefore, we can define a berezinian section $\xi$ on $\Pi T M$ by piecing the locally defined $\xi_{U}$ 's together.

This berezinian section is also a berezinian volume. In fact, this is true locally and, if $U$ and $V$ are open sets such that $U \cap V \neq \emptyset$, and if local forms $\alpha$ and $\beta$ satisfy $\xi_{U} \cdot \alpha=\xi_{V} \cdot \beta$, then $\alpha=\beta$, on $U \cap V$.

Finally, if $\alpha_{(n)}=f \mathrm{~d} x^{1} \wedge \cdots \wedge \mathrm{d} x^{n}$, where $f \in C^{\infty}(U)$, then $\xi_{U}(\alpha)=f \mathrm{~d}^{G} x^{1} \wedge$ $\cdots \wedge \mathrm{d}^{G} x^{n}$, and therefore

$$
\widetilde{\xi_{U}(\alpha)}=f \mathrm{~d}^{G} x^{1} \widetilde{\wedge} \wedge \mathrm{d}^{G} x^{n}=f \mathrm{~d} x^{1} \wedge \cdots \wedge \mathrm{d} x^{n}=\alpha_{(n)},
$$

and $\xi$ satisfies condition (24). 
2.4.2. The canonical divergence operator on $\Pi T M$. Let us denote by $\operatorname{div}_{\text {can }}$ the divergence operator associated to the canonical berezinian volume on ПTM. The graded vector fields on $\Pi T M$ are the derivations of the sheaf of differential forms. Let us denote the sheaf of vector-valued differential $k$-forms by $\Omega^{k}(M ; T M)$, for $k \geq 0$. By the Frölicher-Nijenhuis theorem [9], we know that a derivation $D$ of degree $k$ of $\Omega(M)$ can be uniquely written as

$$
D=\mathcal{L}_{K}+i_{L}
$$

where $K$ is a section of $\Omega^{k}(M ; T M)$ and $L$ is a section of $\Omega^{k+1}(M ; T M)$.

We introduce the notation $\mathcal{C}$ for the $(1,1)$-contraction map from $\Omega^{k}(M ; T M)$ to $\Omega^{k-1}(M)$, defined on a decomposable element $K=\omega \otimes X$, where $X$ is a vector field and $\omega$ is a $k$-form, by $\mathcal{C} K=i_{X} \omega$, for $k \geq 1$, and by $\mathcal{C}=0$ on $\Omega^{0}(M ; T M)$.

Lemma 2.10. For $K$ a section of $\Omega^{k}(M ; T M)$ and $L$ a section of $\Omega^{k+1}(M ; T M)$,

$$
\operatorname{div}_{c a n}\left(i_{L}\right)=(-1)^{k} \mathcal{C} L, \quad \operatorname{div}_{c a n}(\mathrm{~d})=0, \quad \operatorname{div}_{c a n}\left(\mathcal{L}_{K}\right)=-\mathrm{d}(\mathcal{C} K),
$$

where d denotes the de Rham differential.

Proof. We shall first compute $\operatorname{div}_{\text {can }}\left(i_{L}\right)$ for a decomposable $L=\omega \otimes X$ where $\omega$ is a section of $\Omega^{k+1}(M)$. For any differential form $\alpha$,

$$
\begin{aligned}
\widetilde{\left(\mathcal{L}_{i_{L}} \xi\right) \alpha} & =\left(\widehat{\left.\xi \circ i_{L}\right)} \alpha=\widehat{\xi\left(i_{L} \alpha\right)}\right. \\
& =\xi\left(\widetilde{\omega \wedge i_{X} \alpha}\right)=\left(\omega \wedge i_{X} \alpha\right)_{(n)}=\omega \wedge i_{X}\left(\alpha_{(n-k)}\right) \\
& =(-1)^{k} i_{X}(\omega) \wedge \alpha_{(n-k)}=(-1)^{k}\left(i_{X}(\omega) \wedge \alpha\right)_{(n)} \\
& =\xi\left((-1)^{k} i_{X}(\omega) \wedge \alpha\right),
\end{aligned}
$$

where we have used the relation

$$
\left(i_{X} \omega\right) \wedge \alpha_{(n-k)}+(-1)^{k+1} \omega \wedge i_{X} \alpha_{(n-k)}=i_{X}\left(\omega \wedge \alpha_{(n-k)}\right)=0 .
$$

Therefore $\mathcal{L}_{i_{L}} \xi=\xi \cdot\left((-1)^{k} i_{X}(\omega)\right)=\xi \cdot\left((-1)^{k} \mathcal{C} L\right)$, and $\operatorname{div}_{c a n}\left(i_{L}\right)=(-1)^{k} \mathcal{C} L$.

Similarly, for the derivation $d$,

$$
\widetilde{\left(\mathcal{L}_{\mathrm{d}} \xi\right) \alpha}=\widetilde{(\xi \circ \mathrm{d})} \alpha=\widetilde{\xi(\mathrm{d} \alpha)}=\mathrm{d}\left(\alpha_{(n-1)}\right)
$$

which is always an exact form. Thus $\mathcal{L}_{\mathrm{d}} \xi=0$. Finally, using (16), we obtain

$$
\operatorname{div}_{\xi}\left(\mathcal{L}_{K}\right)=\operatorname{div}_{\xi}\left(\left[i_{K}, \mathrm{~d}\right]\right)=i_{K}\left(\operatorname{div}_{\xi}(\mathrm{d})\right)-(-1)^{k-1} \mathrm{~d}\left(\operatorname{div}\left(i_{K}\right)\right)=-\mathrm{d}(\mathcal{C} K) .
$$

In particular, if $X$ is a vector field on $M$, then

$$
\operatorname{div}_{c a n}\left(i_{X}\right)=0, \quad \operatorname{div}_{c a n}\left(\mathcal{L}_{X}\right)=0 .
$$

2.4.3. Generators of the Koszul-Schouten bracket. We shall now assume that the base manifold $M$ is equipped with a Poisson structure. Given a Poisson manifold $(M, P)$, there is an odd Poisson bracket, $\llbracket, \rrbracket_{P}$, on the supermanifold $\Pi T M$, called the Koszul-Schouten bracket, that is characterized by the conditions,

$$
\llbracket f, g \rrbracket_{P}=0, \quad \llbracket f, \mathrm{~d} g \rrbracket_{P}=\{f, g\}, \quad \llbracket \mathrm{d} f, \mathrm{~d} g \rrbracket_{P}=\mathrm{d}\{f, g\},
$$

for all $f, g \in C^{\infty}(M)$, where $\{$,$\} denotes the Poisson bracket on C^{\infty}(M)$ defined by $P$, together with the graded Leibniz rule. It was shown by Koszul [24 that a generator for this bracket is the Poisson homology operator, $\partial_{P}=\left[\mathrm{d}, i_{P}\right]$, sometimes 
called the Koszul-Brylinski operator. See [14, and also [21]. On the other hand, we know that, given a berezinian volume $\xi$ on $\Pi T M$, the operator $\Delta^{(P), \xi}$ defined by

$$
\Delta^{(P), \xi}(\alpha)=(-1)^{|\alpha|} \frac{1}{2} \operatorname{div}_{\xi}\left(\llbracket \alpha, . \rrbracket_{P}\right),
$$

for any differential form $\alpha$, is also a generator of $\llbracket, \rrbracket_{P}$.

Theorem 2.11. The generator $\Delta^{(P), \text { can }}$ of the Koszul-Schouten bracket associated to the canonical berezinian volume coincides with $\partial_{P}$, and $\left(\Delta^{(P), \text { can }}\right)^{2}=0$.

Proof. It suffices to prove that $\Delta^{(P), \text { can }}$ and $\partial_{P}$ agree on 1-forms, and it is enough to show that both vanish on exact 1-forms, $\alpha=\mathrm{d} f$, where $f \in C^{\infty}(M)$. In fact, $\llbracket \mathrm{d} f, . \rrbracket_{P}=\mathcal{L}_{\#_{P} \mathrm{~d} f}$, where $\#_{P} \mathrm{~d} f=\{f,$.$\} . From (25), it follows that$ $\Delta^{(P), c a n}(\mathrm{~d} f)=-\frac{1}{2} \operatorname{div}_{c a n}\left(\mathcal{L}_{\#_{P}} \mathrm{~d} f\right)=0$. And clearly $\partial_{P}(\mathrm{~d} f)=0$. Moreover, $\left(\partial_{P}\right)^{2}=0$, since $\left[\mathrm{d}, \partial_{P}\right]=0$ and $\left[i_{P}, \partial_{P}\right]=0$, and therefore $\left(\Delta^{(P), \text { can }}\right)^{2}=0$.

Remark. Any nondegenerate metric $g$ on the manifold $M$ defines an isomorphism from multivectors to differential forms. Hence, from the Schouten bracket of multivectors, we obtain a $\mathbb{Z}$-graded bracket $\llbracket, \rrbracket_{g}$ on the sheaf of differential forms on $M$. Then, the codifferential $\delta_{g}$ associated to $g$ is a generator of this bracket. (See 440] or [6.).) One can also consider the operator associated to the canonical berezinian on $\Pi T M$, defined by

$$
\Delta^{(g), \text { can }}(\alpha)=(-1)^{|\alpha|} \frac{1}{2} \operatorname{div}_{c a n}\left(\llbracket \alpha, . \rrbracket_{g}\right),
$$

for any differential form $\alpha$, and one can show that these two generators of the bracket $\llbracket, \rrbracket_{g}$ coincide, $\Delta^{(g), c a n}=\delta_{g}$.

2.5. QS, SP and QSP-manifolds. The following definitions, adapted from [37] and [38], will be useful in order to reformulate some of our results.

Definition 2.12. Let $(M, \mathcal{A})$ be a supermanifold, $D$ an odd vector field and $\xi$ a berezinian volume. We say that $((M, \mathcal{A}), D, \xi)$ is a QS-manifold if $D^{2}=0$ and $\operatorname{div}_{\xi}(D)=0$.

Definition 2.13. Let $(M, \mathcal{A})$ be a supermanifold, $\pi$ an odd Poisson bracket and $\xi$ a berezinian volume. We say that $((M, \mathcal{A}), \pi, \xi)$ is a weak SP-manifold if $\left(\Delta^{\pi, \xi}\right)^{2}=0$, where $\Delta^{\pi, \xi}$ is defined by (19). If the Poisson bracket is nondegenerate, then the supermanifold is an SP-manifold.

Definition 2.14. Let $(M, \mathcal{A})$ be a supermanifold, $\pi$ an odd Poisson bracket, $D$ an odd vector field and $\xi$ a berezinian volume. We say that $((M, \mathcal{A}), \pi, D, \xi)$ is a weak QSP-manifold if

- $((M, \mathcal{A}), \pi, \xi)$ is a weak SP-manifold,

- $((M, \mathcal{A}), D, \xi)$ is a $Q S$-manifold, and

- $D$ is a derivation of the odd Poisson bracket, $\pi$.

If the Poisson bracket is nondegenerate, and if $D$ is the hamiltonian vector field defined by an even section of $\mathcal{A}$, then the supermanifold is a QSP-manifold. 
If the graded vector field $D$ is the hamiltonian vector field $\llbracket h, . \rrbracket$, where $h$ is an even section of $\mathcal{A}$, then $D^{2}=0$ if and only if $\llbracket h, h \rrbracket=0$. In field theory, this condition appears under the name classical master equation.

It follows from identity (3) that, if $\Delta$ is a generator of the odd Poisson bracket $\llbracket, \rrbracket$ and if $D$ is a graded vector field on $(M, \mathcal{A})$, a necessary and sufficient condition for $D$ to be a derivation of the odd Poisson bracket is that the graded commutator, $[D, \Delta]$, be a derivation of the associative multiplication of $\mathcal{A}$. This implies

Proposition 2.15. If $\pi$ and $\xi$ define a weak SP-structure on $(M, \mathcal{A})$, if $D$ and $\xi$ define a $Q S$-structure on $(M, \mathcal{A})$, and if $[D, \Delta]=0$, then $\pi, D$ and $\xi$ define a weak QSP-structure on $(M, \mathcal{A})$.

The following theorems follow in part from the results of Section 2.3 and 2.4. See also [1].

Theorem 2.16. (i) For any manifold $M$ with a volume element, the supermanifold $\Pi T^{*} M$, with the Schouten bracket and the berezinian volume $\xi_{\mu}$, is an SP-manifold.

(ii) Let $(M, P)$ be a Poisson manifold, and let $d_{P}=\llbracket P$, . $\rrbracket$ be the LichnerowiczPoisson differential. Then $\Pi T^{*} M$, with the Schouten bracket, the odd vector field $d_{P}$, and the canonical berezinian volume, is a QSP-manifold.

Proof. In fact, the odd vector field $d_{P}$ is of square 0 , because $\llbracket P, P \rrbracket=0$, and it is a derivation of the Schouten bracket by the graded Jacobi identity.

Theorem 2.17. (i) For any manifold $M$, the supermanifold $\Pi T M$, with the de Rham differential $\mathrm{d}$ and the canonical berezinian volume, is a QS-manifold.

(ii) Let $(M, P)$ be a Poisson manifold, and let $\llbracket, \rrbracket_{P}$ be the Koszul-Schouten bracket. Then $\Pi T M$ with the odd Poisson bracket $\llbracket, \rrbracket_{P}$, the de Rham differential $\mathrm{d}$ and the canonical berezinian volume, is a weak QSP-manifold. If $P$ is a nondegenerate Poisson structure, then ПTM is a QSP-manifold.

Proof. To complete the proof, we only have to recall that the de Rham differential $\mathrm{d}$ is a derivation of $\llbracket, \rrbracket_{P}$, and that, when $P$ is nondegenerate with inverse the symplectic form, $\omega$, then $\mathrm{d}=\llbracket \omega, . \rrbracket_{P}$, so that $\mathrm{d}$ is the hamiltonian vector field associated to $\omega$. (See [22] or [5] for a proof of this fact.)

\section{Linear CONNECTIONS AND Generators of ODD POISSON BRACKETS}

Divergence operators on smooth manifolds can be defined not only by means of volume forms, but also by means of connections. (See [15].) While in Section 2, we generalized the first approach to supermanifolds, replacing volume forms by their graded analogue, the berezinian volumes, in this section we generalize the second method, defining divergence operators by means of graded connections.

3.1. Divergence operators defined by graded connections. We first recall the notion of graded connection. See [30] and [31] for the definitions of left and right graded connections. Here we consider only left graded connections, which we

simply call connections. Let $(M, \mathcal{A})$ be a supermanifold and let Der $\mathcal{A}$ be the sheaf of derivations of $\mathcal{A}$. 
Definition 3.1. Let $\mathcal{S}$ be a sheaf of $\mathcal{A}$-modules on $M$. A left graded connection, or simply a connection, on $\mathcal{S}$ is a morphism of sheaves of graded vector spaces from Der $\mathcal{A} \otimes \mathcal{S}$ to $\mathcal{S}$, denoted $D \otimes \alpha \mapsto \mathbb{\nabla}_{D} \alpha$, which satisfies the identity

$$
\mathbb{\nabla}_{f D} \alpha=f \mathbb{\nabla}_{D} \alpha
$$

and the Leibniz rule,

$$
\mathbb{\nabla}_{D}(f \alpha)=D(f) \alpha+(-1)^{|D||f|} f \mathbb{\nabla}_{D} \alpha,
$$

for any section $f$ of $\mathcal{A}$, any derivation $D$ of $\mathcal{A}$, and any section $\alpha$ of $\mathcal{S}$.

$A$ connection on the sheaf Der $\mathcal{A}$ of derivations of $\mathcal{A}$ is a graded linear connection or simply a linear connection on $(M, \mathcal{A})$.

Definition 3.2. The curvature, $R^{\mathbb{\nabla}}$, of a connection, $\mathbb{\nabla}$, on $\mathcal{S}$ is defined by

$$
R^{\mathbb{\nabla}}\left(D_{1}, D_{2}\right)=\left[\mathbb{\nabla}_{D_{1}}, \mathbb{\nabla}_{D_{2}}\right]-\mathbb{\nabla}_{\left[D_{1}, D_{2}\right]},
$$

for any derivations $D_{1}, D_{2}$ of $\mathcal{A}$.

The torsion, $T^{\mathbb{\nabla}}$, of a linear connection, $\mathbb{\nabla}$, on $(M, \mathcal{A})$ is defined by

$$
T^{\mathbb{\nabla}}\left(D_{1}, D_{2}\right)=\mathbb{\nabla}_{D_{1}} D_{2}-(-1)^{\left|D_{1}\right|\left|D_{2}\right|} \mathbb{\nabla}_{D_{2}} D_{1}-\left[D_{1}, D_{2}\right] .
$$

We shall now define the divergence operator associated with a linear connection on $(M, \mathcal{A})$. Let $s \operatorname{Tr}$ denote the supertrace of an endomorphism of sheaves of $\mathcal{A}$ modules (see, e.g., [30 or [7]), and let $\operatorname{ad}_{D}$ denote the endomorphism of Der $\mathcal{A}$, $E \mapsto[D, E]$. For any graded vector field $D$, we set

$$
\operatorname{div}_{\mathbb{\nabla}}(D)=s \operatorname{Tr}\left(\mathbb{\nabla}_{D}-\operatorname{ad}_{D}\right) .
$$

Proposition 3.3. For any linear connection, $\mathbb{\nabla}$, on $(M, \mathcal{A})$, the $m a p$, $\operatorname{div}_{\mathbb{\nabla}}$ : Der $\mathcal{A} \rightarrow \mathcal{A}$, defined by (26) is a divergence operator.

Proof. The map $\operatorname{div}_{\mathbb{\nabla}}$ is even. It follows from $[f D, E]=f[D, E]-(-1)^{(|f|+|D|)|E|} E(f) D$, that

$$
\begin{aligned}
\operatorname{div}_{\mathbb{\nabla}}(f D) & =s \operatorname{Tr}\left(\mathbb{\nabla}_{f D}-\operatorname{ad}_{f D}\right) \\
& =f \operatorname{div}_{\mathbb{\nabla}}(D)+s \operatorname{Tr}\left(E \mapsto(-1)^{(|f|+|D|)|E|} E(f) D\right) .
\end{aligned}
$$

Let the graded dimension of the supermanifold be $m \mid n$, and let us choose a system of local graded coordinates $\left(x^{1}, \ldots, x^{m}, s^{1}, \ldots, s^{n}\right)$. We find that

$$
\begin{aligned}
s \operatorname{Tr}(E & \left.\mapsto(-1)^{(|f|+|D|)|E|} E(f) D\right)=\sum_{i=1}^{m} \frac{\partial f}{\partial x^{i}} D\left(x^{i}\right)-(-1)^{|f|+|D|} \sum_{\rho=1}^{n} \frac{\partial f}{\partial s^{\rho}} D\left(s^{\rho}\right) \\
& =(-1)^{|f||D|} \sum_{i=1}^{m} D\left(x^{i}\right) \frac{\partial f}{\partial x^{i}}+(-1)^{|f||D|} \sum_{\rho=1}^{n} D\left(s^{\rho}\right) \frac{\partial f}{\partial s^{\rho}}=(-1)^{|f||D|} D(f),
\end{aligned}
$$

where we have used the local expression of the derivation $D$ in the basis of local graded vector fields, $\left(\frac{\partial}{\partial x^{1}}, \ldots, \frac{\partial}{\partial x^{m}}, \frac{\partial}{\partial s^{1}}, \ldots, \frac{\partial}{\partial s^{n}}\right)$.

Proposition 3.4. Let $\mathbb{\nabla}$ be a torsionless linear connection on $\mathcal{A}$ and let $D_{1}$ and $D_{2}$ be graded vector fields. Then

$$
\mathcal{R}^{\operatorname{div}_{\nabla}}\left(D_{1}, D_{2}\right)=-s \operatorname{Tr}\left(R^{\mathbb{\nabla}}\left(D_{1}, D_{2}\right)\right) .
$$


Proof. We have to prove that

$$
\begin{aligned}
D_{1}\left(s \operatorname{Tr}\left(\mathbb{\nabla}_{D_{2}}-\operatorname{ad}_{D_{2}}\right)\right) & -(-1)^{\left|D_{1}\right|\left|D_{2}\right|} D_{2}\left(s \operatorname{Tr}\left(\mathbb{\nabla}_{D_{1}}-\operatorname{ad}_{D_{1}}\right)\right)-s \operatorname{Tr}\left(\mathbb{\nabla}_{\left[D_{1}, D_{2}\right]}-\operatorname{ad}_{\left[D_{1}, D_{2}\right]}\right) \\
& =s \operatorname{si}\left(\left[\mathbb{\nabla}_{D_{1}}, \mathbb{\nabla}_{D_{2}}\right]-\mathbb{\nabla}_{\left[D_{1}, D_{2}\right]}\right) .
\end{aligned}
$$

This result follows from a computation of these two expressions in local coordinates, for pairs of commuting graded vector fields, $D_{1}$ and $D_{2}$, in a local basis.

3.2. Generators defined by graded connections. We now assume that $(M, \mathcal{A})$ has an odd Poisson structure, $\pi$, whose bracket we denote by $\llbracket, \rrbracket$. Let $\mathbb{\nabla}$ be a linear connection on $(M, \mathcal{A})$. Following the general pattern of Section [1.3, we define the operator $\Delta^{\pi, \mathbb{}}: \mathcal{A} \rightarrow \mathcal{A}$ by

$$
\Delta^{\pi, \mathbb{}}(f)=(-1)^{|f|} \frac{1}{2} \operatorname{div}_{\mathbb{\nabla}}(\llbracket f, . \rrbracket),
$$

for any section $f$ of $\mathcal{A}$. It follows from Proposition 3.3 and Theorem 1.2 that the odd operator $\Delta^{\pi, \mathbb{}}$ is a generator of bracket $\pi$. Therefore, to any linear connection on an odd Poisson manifold, there corresponds a generator of the odd Poisson bracket.

Proposition 3.5. Let $\mathbb{\nabla}$ be a torsionless linear connection on $\mathcal{A}$. The following properties are equivalent

- $\Delta^{\pi, \mathbb{\nabla}}$ is a derivation of the odd Poisson bracket, $\pi$,

- $\left(\Delta^{\pi, \mathbb{\nabla}}\right)^{2}$ is a derivation of the sheaf of associative algebras, $\mathcal{A}$,

- $s \operatorname{Tr}\left(R^{\mathbb{\nabla}}\right)$ vanishes on the sheaf of hamiltonian derivations.

Proof. These equivalences follow from Lemma 1.1, and from Corollary 1.4 together with Proposition 3.4.

We now compare the generators associated to torsionless linear connections, $\mathbb{\nabla}$ and $\mathbb{\nabla}^{\prime}$, on $\mathcal{A}$. The difference $\mathbb{\nabla}_{D}^{\prime}-\mathbb{\nabla}_{D}$ is then a morphism of sheaves of $\mathcal{A}$-modules from Der $\mathcal{A}$ to itself, which we denote by $u(D)$.

Proposition 3.6. Let $\mathbb{\nabla}$ and $\mathbb{W}^{\prime}$ be torsionless linear connections on $\mathcal{A}$. Then

$$
\Delta^{\pi, \mathbb{\nabla}^{\prime}}(f)=\Delta^{\pi, \mathbb{\nabla}}(f)+(-1)^{|f|} \frac{1}{2} s \operatorname{Tr}\left(u\left(X_{f}^{\pi}\right)\right)
$$

for any section $f$ of $\mathcal{A}$.

Proof. This relation follows from the fact that, for any derivation $D$ of $\mathcal{A}, \operatorname{div}_{\mathbb{\nabla}^{\prime}}(D)=$ $\operatorname{div}_{\mathbb{\nabla}}(D)+s \operatorname{Tr}(u(D))$.

Remark. In the case of an ordinary manifold, the trace of the curvature of a linear connection is the curvature of the connection induced on the bundle of top-degree forms. It would be interesting to interpret the supertrace of the curvature of a graded linear connection as the curvature of a connection on the sections of the berezinian sheaf.

3.3. Metrics and metric connections on supermanifolds. We recall the definitions of metrics and metric linear connections on supermanifolds.

Definition 3.7. A graded metric, or simply a metric, on $(M, \mathcal{A})$ is a morphism of sheaves of $\mathcal{A}$-modules, $\langle\rangle:$, Der $\mathcal{A} \otimes \operatorname{Der} \mathcal{A} \rightarrow \mathcal{A}$, such that

- $\left\langle D_{1}, D_{2}\right\rangle=(-1)^{\left|D_{1}\right|\left|D_{2}\right|}\left\langle D_{2}, D_{1}\right\rangle$, for derivations $D_{1}$ and $D_{2} \quad$ (graded symmetry), 
- the map $D \mapsto\langle D, \cdot\rangle$ is an isomorphism of sheaves of $\mathcal{A}$-modules from Der $\mathcal{A}$ to $\operatorname{Hom}_{\mathcal{A}}(\operatorname{Der} \mathcal{A}, \mathcal{A}) \quad$ (nondegeneracy).

Definition 3.8. A linear connection $\mathbb{\nabla}$ on $\mathcal{A}$ is metric with respect to a metric $\langle$, if, for any derivations $D, D_{1}$, and $D_{2}$ of $\mathcal{A}$,

$$
D\left\langle D_{1}, D_{2}\right\rangle=\left\langle\mathbb{\nabla}_{D} D_{1}, D_{2}\right\rangle+(-1)^{\left|D_{1}\right||D|}\left\langle D_{1}, \mathbb{\nabla}_{D}^{0} D_{2}\right\rangle+(-1)^{\left|D_{1}\right|(|D|+1)}\left\langle D_{1}, \mathbb{\nabla}_{D}^{1} D_{2}\right\rangle,
$$

where $\mathbb{\nabla}=\mathbb{\nabla}^{0}+\mathbb{\nabla}^{1}$ is the decomposition of the linear connection into its even and odd components.

The proof of the following theorem, can be found in [27], p. 134, and in [33].

Theorem 3.9. There exists a unique torsionless linear connection which is metric with respect to a given metric. It is determined by

$$
\begin{aligned}
2\left\langle\mathbb{W}_{D_{1}} D_{2}, D_{3}\right\rangle & =D_{1}\left\langle D_{2}, D_{3}\right\rangle+\left\langle\left[D_{1}, D_{2}\right], D_{3}\right\rangle \\
& +(-1)^{\left|D_{1}\right|\left(\left|D_{2}\right|+\left|D_{3}\right|\right)}\left(D_{2}\left\langle D_{3}, D_{1}\right\rangle-\left\langle\left[D_{2}, D_{3}\right], D_{1}\right\rangle\right) \\
& -(-1)^{\left|D_{3}\right|\left(\left|D_{1}\right|+\left|D_{2}\right|\right)}\left(D_{3}\left\langle D_{1}, D_{2}\right\rangle-\left\langle\left[D_{3}, D_{1}\right], D_{2}\right\rangle\right) .
\end{aligned}
$$

The linear connection defined in Theorem 3.9 is called the graded Levi-Civita connection or simply, the Levi-Civita connection of the metric $\langle$,$\rangle . The Levi-Civita$ connection of a homogeneous metric is even. (See also [8], where an expression in local coordinates of the Levi-Civita connection is given in the case of a homogeneous, even metric.)

3.4. Linear connections and Schouten bracket. We shall again consider the supermanifold $\Pi T^{*} M$, whose sheaf of functions is the sheaf of multivectors on $M$. We shall use the notations $f, g \ldots$ for functions on $M$ or on an open set of $M$, and the notations $X, Y, \ldots$ for vector fields and $\alpha, \beta \ldots$ for differential 1-forms.

3.4.1. Graded vector fields on $\Pi T^{*} M$. Whereas the derivations of the algebra of forms on a manifold can be classified by the Frölicher-Nijenhuis theorem [9] (and see Section 2.4.2), the classification of the derivations of the algebra of multivectors on $M$ requires the use of an auxiliary linear connection, $\nabla$. Let $U$ be an open set of $M$. If $K=Q \otimes X$ is a vector-valued multivector on $U$, where $Q$ is a multivector and $X$ is a vector, we define $\nabla_{K} V=Q \wedge \nabla_{X} V$, for any multivector $V$ on $U$. If $L=W \otimes \alpha$ is a 1 -form-valued multivector on $U$, where $W$ is a multivector and $\alpha$ is a differential 1-form, we define $i_{L} V=W \wedge i_{\alpha} V$.

Proposition 3.10. [32] Let $D$ be a graded vector field of degree $r$ on $\Pi T^{*} M$, i.e., a derivation of degree $r$ of the sheaf of multivectors on $M$. On any open set $U$ of $M$, there exist a vector-valued $r$-vector, $K$, and a 1 -form-valued $(r+1)$-vector, $L$, each uniquely defined, such that $\left.D\right|_{U}=\nabla_{K}+i_{L}$.

As a consequence, we see that, if $\left(e_{1}, \ldots, e_{n}\right)$ is a local basis of vector fields on $U$ and $\left(\epsilon^{1}, \ldots, \epsilon^{n}\right)$ is the dual basis, then $\left(\nabla_{e_{1}}, \ldots, \nabla_{e_{n}}, i_{\epsilon^{1}}, \ldots, i_{\epsilon^{n}}\right)$ generate the derivations of the algebra of multivectors over $U$, as a module over the algebra of multivectors over $U$. 
3.4.2. The graded connection on $\Pi T^{*} M$ associated to a linear connection on $M$. We shall show how to associate a metric on $\Pi T^{*} M$ to a linear connection on $M$, and we shall study the Levi-Civita connection of this metric.

Definition 3.11. Let $\nabla$ be a linear connection on $M$. We define a metric $\langle,\rangle_{\nabla}$ on $\Pi T^{*} M$ by its value on derivations of type $\nabla_{X}$, where $X$ is a vector field, and of type $i_{\alpha}$, where $\alpha$ is a 1-form,

$$
\left\langle\nabla_{X}, \nabla_{Y}\right\rangle_{\nabla}=0, \quad\left\langle\nabla_{X}, i_{\alpha}\right\rangle_{\nabla}=\alpha(X), \quad\left\langle i_{\alpha}, i_{\beta}\right\rangle_{\nabla}=0
$$

To verify the nondegeneracy of the metric thus defined, we observe that, in the local basis of derivations $\left(\nabla_{e_{1}}, \ldots, \nabla_{e_{n}}, i_{\epsilon^{1}}, \ldots, i_{\epsilon^{n}}\right)$, the matrix of this metric is $\left(\begin{array}{cc}0 & I d \\ I d & 0\end{array}\right)$. This metric is odd.

Proposition 3.12. Let $\nabla$ be a torsionless linear connection on $M$. The Levi-Civita connection, $\mathbb{\nabla}$, of the metric $\langle,\rangle_{\nabla}$ on $\Pi T^{*} M$ is given by

$$
\mathbb{\nabla}_{\nabla_{X}} \nabla_{Y}=\nabla_{\nabla_{X} Y}+i_{R(\cdot, Y) X}, \quad \mathbb{\nabla}_{\nabla_{X}} i_{\alpha}=i_{\nabla_{X} \alpha}, \quad \mathbb{\nabla}_{i_{\alpha}}=0
$$

where $R$ denotes the curvature tensor of $\nabla$.

Proof. We shall make use of the commutation relations

$$
\left[\nabla_{X}, \nabla_{Y}\right]=\nabla_{[X, Y]}+i_{R(X, Y)}, \quad\left[\nabla_{X}, i_{\alpha}\right]=i_{\nabla_{X} \alpha}, \text { and }\left[i_{\alpha}, i_{\beta}\right]=0
$$

Using Theorem 3.9, Definition 3.11 and the fact that the connection $\nabla$ is torsionless, we obtain

$$
\begin{aligned}
\left\langle\mathbb{\nabla}_{\nabla_{X}} \nabla_{Y}, \nabla_{Z}\right\rangle_{\nabla} & =\frac{1}{2}(R(X, Y) Z-R(Y, Z) X+R(Z, X) Y) \\
& =R(Z, Y) X=\left\langle i_{R(., Y) X}, \nabla_{Z}\right\rangle_{\nabla}, \\
\left\langle\mathbb{\nabla}_{\nabla_{X}} \nabla_{Y}, i_{\alpha}\right\rangle_{\nabla} & =\alpha\left(\nabla_{X} Y\right)=\left\langle\nabla_{\nabla_{X} Y}, i_{\alpha}\right\rangle_{\nabla},
\end{aligned}
$$

and

$$
\left\langle\mathbb{\nabla}_{\nabla_{X}} i_{\alpha}, \nabla_{Y}\right\rangle_{\nabla}=\left(\nabla_{X} \alpha\right) Y=\left\langle i_{\nabla_{X} \alpha}, \nabla_{Y}\right\rangle_{\nabla}, \quad\left\langle\mathbb{\nabla}_{\nabla_{X}} i_{\alpha}, i_{\beta}\right\rangle_{\nabla}=0
$$

From these relations and the nondegeneracy of the graded metric we obtain the first two formulæ, while the third follows from the fact that $\mathbb{W}$ is torsionless.

Proposition 3.13. The curvature $R^{\mathbb{V}}$ of the Levi-Civita connection, $\mathbb{\nabla}$, of the metric $\langle,\rangle_{\nabla}$ on $\Pi T^{*} M$, satisfies

$$
\begin{aligned}
R^{\nabla}\left(\nabla_{X}, \nabla_{Y}\right) \nabla_{Z} & =\nabla_{R(X, Y) Z}+i_{\left(\nabla_{X} R\right)(\cdot, Z) Y}+i_{\left(\nabla_{Y} R\right)(\cdot, Z) X}, \\
R^{\mathbb{\nabla}}\left(\nabla_{X}, \nabla_{Y}\right) i_{\alpha} & =i_{R(X, Y)^{*} \alpha},
\end{aligned}
$$

where $R$ denotes the curvature tensor of $\nabla$, and $R(X, Y)^{*}$ denotes the transpose of $R(X, Y)$. Moreover $R^{\mathbb{\nabla}}\left(\nabla_{X}, i_{\alpha}\right)=R^{\mathbb{\nabla}}\left(i_{\alpha}, i_{\beta}\right)=0$.

Proof. The proof is a straightforward computation using Proposition 3.12 .

Corollary 3.14. Let $\nabla$ be a torsionless linear connection on $M$. Then the LeviCivita connection of the metric $\langle,\rangle_{\nabla}$ on $\Pi T^{*} M$ is flat if and only if $\nabla$ is flat. 
3.4.3. Generators of the Schouten bracket. We have just seen that, to a torsionless linear connection $\nabla$ on $M$ we can associate the Levi-Civita connection, $\mathbb{\nabla}$, of the odd metric $\langle,\rangle_{\nabla}$ on $\Pi T^{*} M$, and therefore, by (28), a generator of the Schouten bracket, which we shall denote by $\Delta^{\text {Schouten, } \mathbb{\nabla}}$. There exists another construction, due to Koszul [24, which associates a generator of the Schouten bracket to a torsionless linear connection $\nabla$ on $M$. To $\nabla$, he first associates the corresponding divergence operator, defined on vector fields $X$ by

$$
\operatorname{div}_{\nabla}(X)=\operatorname{Tr}\left(\nabla_{X}-\operatorname{ad}_{X}\right) .
$$

This is the definition that is used in fact in [24] (although it appears by mistake with the opposite sign in its first occurrence, page 262, before Lemma (2.1)). This map is a divergence operator, $i$. e., satisfies (4), on the purely even algebra $C^{\infty}(M)$. For a flat connection on flat space, it reduces to the elementary divergence. He then shows, using a local basis of vector fields, that there is a unique operator on the multivectors, $\Delta^{\nabla}$, of degree -1 , that extends the operator - $\operatorname{div}_{\nabla}$ and generates the Schouten bracket. We shall now show that the generators of the Schouten bracket obtained by these two constructions coincide.

Lemma 3.15. For any vector field $X$, and for any 1 -form $\alpha$,

$$
\operatorname{div}_{\mathbb{\nabla}}\left(\nabla_{X}\right)=\operatorname{div}_{\nabla}(X), \quad \operatorname{div}_{\mathbb{\nabla}}\left(i_{\alpha}\right)=0 .
$$

Proof. If $\left(x^{1}, \ldots, x^{n}\right)$ is a system of local coordinates on $M$, then a local basis of graded derivations on $\Pi T^{*} M$ is $\left(\nabla_{\frac{\partial}{\partial x^{1}}}, \ldots, \nabla_{\frac{\partial}{\partial x^{n}}}, i_{\mathrm{d} x^{1}}, \ldots, i_{\mathrm{d} x^{n}}\right)$. We use the relations $<\nabla_{\frac{\partial}{\partial x^{j}}}, \mathrm{~d}^{G} x^{k}>=\delta_{j}^{k}$, and $<i_{\mathrm{d} x^{j}}, \mathrm{~d}^{G} x^{k}>=0$. In order to compute $\operatorname{div}_{\mathbb{\nabla}}\left(\nabla_{X}\right)=s \operatorname{Tr}\left(\mathbb{\nabla}_{\nabla_{X}}-\operatorname{ad}_{\nabla_{X}}\right)$, we first observe that, because $\mathbb{W}$ is torsionless,

$$
\mathbb{\nabla}_{\nabla_{X}} i_{\mathrm{d} x^{j}}-\left[\nabla_{X}, i_{\mathrm{d} x^{j}}\right]=\mathbb{\nabla}_{i_{\mathrm{d} x^{j}}} \nabla_{X}=0
$$

Therefore

$$
\begin{aligned}
& s \operatorname{si}\left(\mathbb{\nabla}_{\nabla_{X}}-\operatorname{ad}_{\nabla_{X}}\right)=\sum_{j=1}^{n}<\mathbb{V}_{\nabla_{X}} \nabla_{\frac{\partial}{\partial x^{j}}}-\left[\nabla_{X}, \nabla_{\frac{\partial}{\partial x^{j}}}\right], \mathrm{d}^{G} x^{j}> \\
& =\sum_{j=1}^{n}<\mathbb{\nabla}_{\frac{\partial}{\partial x^{j}}} \nabla_{X}, \mathrm{~d}^{G} x^{j}>=\sum_{j=1}^{n}<\nabla_{\frac{\partial}{\partial x^{j}}} X, \mathrm{~d} x^{j}>=\operatorname{div}_{\nabla}(X) .
\end{aligned}
$$

Theorem 3.16. For any torsionless linear connection $\nabla$ on $M$, the generators

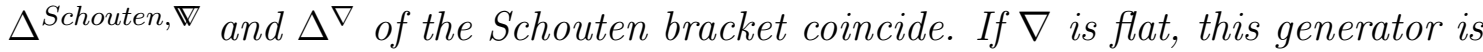
of square 0 .

Proof. Since we know that both operators are generators of the Schouten bracket, we need only show that they coincide on functions and on vector fields. On functions, $\Delta^{\text {Schouten }, \mathbb{\nabla}}$ vanishes since $\llbracket f, \cdot \rrbracket=i_{d f}$ and $\operatorname{div}_{\mathbb{\nabla}}\left(i_{d f}\right)=0$, as does $\Delta^{\nabla}$ because it is of degree -1 . Now, for any vector field $X$ and any torsionless linear connection $\nabla$ on an open set $U$ of $M, \llbracket X, . \rrbracket=\nabla_{X}-i_{\nabla X}$, since both derivations of the sheaf of multivectors coincide on functions and on vectors. If $\left(e_{1}, \ldots, e_{n}\right)$ is a local basis of vector fields on $U$ and $\left(\epsilon^{1}, \ldots, \epsilon^{n}\right)$ is the dual basis, then the 1 -form-valued vector 
$\nabla X$ in $U$ can be written as $\sum_{j=1}^{n} \nabla_{e_{j}} X \otimes \epsilon^{j}$. Thus $i_{\nabla X}=\nabla_{e_{j}} X \wedge i_{\epsilon^{j}}$. Therefore, by Proposition 3.3 and Lemma 3.15 ,

$$
\begin{aligned}
\operatorname{div}_{\nabla}\left(i_{\nabla X}\right) & =\operatorname{div}_{\nabla}\left(\sum_{j=1}^{n} \nabla_{e_{j}} X \wedge i_{\epsilon^{j}}\right) \\
& =\sum_{j=1}^{n} \nabla_{e_{j}} X \operatorname{div}_{\nabla}\left(i_{\epsilon^{j}}\right)-\sum_{j=1}^{n} \epsilon^{j}\left(\nabla_{e_{j}} X\right)=-\operatorname{div}_{\nabla}(X) .
\end{aligned}
$$

It follows that

$$
\Delta^{\text {Schouten }, \mathbb{\nabla}}(X)=-\frac{1}{2} \operatorname{div}_{\mathbb{\nabla}}(\llbracket X, \cdot \rrbracket)=-\operatorname{div}_{\nabla}(X) .
$$

It follows from Corollaries 1.4 and 3.14 together with Proposition 3.4 that, if $\nabla$ is flat, the operator $\left(\Delta^{\text {Schouten }, \mathbb{\nabla}}\right)^{2}$ is a derivation of the sheaf of multivectors with respect to the exterior product. Since, moreover, $\left(\Delta^{\text {Schouten }, \mathbb{\nabla}}\right)^{2}$ is of $\mathbb{Z}$-degree -2 , it vanishes.

Remark. Koszul [24] proves that, conversely, any generator of the Schouten bracket of multivectors on a manifold $M$ is of the form $\Delta^{\nabla}$ for some torsionless linear connection on $M$, and that two connections give rise to the same generator of the Schouten bracket if and only if they induce the same linear connection on $\bigwedge^{m} T M, m$ being the dimension of the manifold. We have not found any straightforward extension of this result to the case of odd Poisson brackets on supermanifolds in general.

3.4.4. Conclusion. Given a smooth manifold $M$, we set $A=C^{\infty}(M)$, and we let Der $A$ denote the module of vector fields on $M$. The definition of a divergence operator on a graded algebra reduces, in the purely even case of $A$, to the requirement that the linear operator, div : Der $A \rightarrow A$, satisfy the identity $\operatorname{div}(f X)=f \operatorname{div} X+X(f)$, for any $f \in A$ and $X \in \operatorname{Der} A$. The operators $\operatorname{div}_{\nabla}$, considered in Section 3.4.3, where $\nabla$ are torsionless linear connections on $M$, are examples of divergence operators. Other examples are furnished by the operators $\operatorname{div}_{\mu}$ associated to volume forms, $\mu$, on an orientable manifold $M$. The Schouten bracket is an odd Poisson bracket on the graded commutative, associative algebra, $\boldsymbol{\Lambda}=\bigwedge_{A}($ Der $A)=\oplus_{k=0}^{m} \bigwedge_{A}^{k}($ Der $A)$, where $m$ is the dimension of the manifold $M$. It is the opposite of a divergence operator that can be extended into a generator of the Schouten bracket. In fact, for any divergence operator on $A$, the operator - div can be uniquely extended to a generator of $\mathbb{Z}$-degree -1 , denoted $\Delta^{\text {(div) }}$, of the Schouten bracket. One can characterize the generator $\Delta^{(\text {div })}$ recursively since, for any $f \in A$, it commutes with the interior product $i_{\mathrm{d} f}$. More generally, for any form $\alpha$,

$$
\left[i_{\alpha}, \Delta^{(\operatorname{div})}\right]=-i_{\mathrm{d} \alpha}
$$

It is easy to see that, in the purely even case, a divergence operator $\operatorname{div}: \operatorname{Der} A \rightarrow$ $A$ is nothing but a right $(A$, Der $A)$-connection on $A$, in the sense of Huebschmann [15] [16]. In fact, if div is a divergence operator, then

$$
(f, X) \in A \times \operatorname{Der} A \mapsto f \circ X=-\operatorname{div}(f X) \in A
$$

is a right $(A, \operatorname{Der} A)$-connection on $A$, and, conversely, if $(f, X) \in A \times \operatorname{Der} A \mapsto$ $f \circ X \in A$ is a right $(A$, Der $A$ )-connection on $A$, then $X \in \operatorname{Der} A \mapsto-1 \circ X$, where 1 is the unit of $A$, is a divergence operator. Moreover, the right $(A, \operatorname{Der} A)$ connection is a right $(A$, Der $A)$-module structure if and only if the curvature of the divergence operator, defined by (5), vanishes. In fact,

$$
\left(f \circ X_{1}\right) \circ X_{2}-\left(f \circ X_{2}\right) \circ X_{1}-f \circ\left[X_{1}, X_{2}\right]=f \mathcal{R}^{\operatorname{div}}\left(X_{1}, X_{2}\right) \text {. }
$$


In the papers cited above, the notion of a divergence operator does not appear explictly, but the preceding remarks show that the 1-to-1 correspondence ([15], Theorem 1) between right $(A, \operatorname{Der} A)$-connections on $A$ and generating operators of the Schouten bracket of $\boldsymbol{\Lambda}$ yields a 1-to-1 correspondence between divergence operators and generators, which restricts to a 1 -to- 1 correspondence between divergence operators whose curvature vanishes and generators whose square vanishes. Also, the 1-to-1 correspondence (\|15], Theorem 3) between right $(A$, Der $A)$-connections on $A$ and left $(A$, Der $A)$-connections on the top exterior power, $\bigwedge_{A}^{m} \operatorname{Der} A=\bigwedge_{A}^{\text {top }} \operatorname{Der} A$, translates into a 1-to-1 correspondence between divergence operators and left $(A, \operatorname{Der} A)$ connections on the top exterior power. The canonical bundle $\bigwedge_{A}^{\text {top }} \operatorname{Der} A$ is, in a natural way, a right module; equipping it with a left module structure, which can be done by choosing a volume element, is equivalent to equipping $A$ itself with a right module structure and therefore to selecting a divergence operator whose curvature vanishes (cf Propositon 2.3). To summarize, divergence operators, right connections on $A$, left connections on the top exterior power of Der $A$, and generators of the Schouten bracket are in 1-to-1 correspondence. The definition of divergence operators and the preceding constructions extend to the framework of Lie algebroids and to that of Lie-Rinehart algebras. See [15], [16] and also [44] and [21]. While there is a functor from Lie-Rinehart algebras to Gerstenhaber algebras, there is also a functor from Lie-Rinehart algebras with a divergence operator (resp., divergence operator with vanishing curvature) to Gerstenhaber algebras with a generator (resp., to Batalin-Vilkovisky algebras). In the case of a complex analytic manifold $M$ and its algebra of analytic functions, the left $(A$, Der $A)$-module structures on the canonical bundle (top exterior power of holomorphic vector fields) are called Calabi-Yau structures [36. In this case, left (resp., right) ( $A$, Der $A$ )-module structures coincide with left (resp., right) $\mathcal{D}_{M}$-module structures.

The extension of the above 1-to-1 correspondences valid in the purely even case to the case where $A$ itself is a $\mathbb{Z}_{\text {- or }} \mathbb{Z}_{2}$-graded algebra, $\mathbf{A}$, remains to be done. The appropriate framework is that of the graded Lie-Rinehart algebras, whose theory has already been developped by Huebschmann (1990, unpublished), and left and right (A, Der A)-connections and module structures in an appropriate sense. Sheaves of graded Lie-Rinehart algebras should then be considered, the fundamental example being $(\mathcal{A}$, Der $\mathcal{A})$, for any supermanifold $(M, \mathcal{A})$. A divergence operator with vanishing curvature should define a right $(\mathcal{A}$, Der $\mathcal{A})$-module structure on $\mathcal{A}$, and there should be 1-to-1 correspondences between divergence operators, right structures on the structural sheaf and left structures on the berezinian sheaf. Another approach is by means of the theory of $\mathcal{D}$-modules. Left and right $\mathcal{D}$-modules on complex supermanifolds have been studied by Penkov [34], who showed that the berezinian sheaf of a complex analytic supermanifold is a right $\mathcal{D}$-module in a canonical way. Defining a left $\mathcal{D}$-module structure on the berezinian sheaf, which can be done by choosing a berezinian volume, is equivalent to defining a right $\mathcal{D}$-module structure on the structural sheaf, and should be equivalent to the choice of a divergence operator.

One can define graded analogues of the modules of multivectors on a manifold as modules of skew-symmetric multiderivations of $\mathbf{A}$, and one can generalize these notions to the case of sheaves of graded algebras over a manifold. Multigraded generalizations of the Schouten bracket on the space of skew-symmetric multiderivations 
of a graded algebra were defined by Krasil'shchik in [26], following his earlier paper [25]. An analogue of the 1-to-1 correspondence between divergence operators and generators should be also valid in the graded case.

Conjecture. A divergence operator on the graded algebra A, up to sign factors, can be uniquely extended to an operator on the skew-symmetric multiderivations of $\mathbf{A}$ that generates, in a suitable sense, the bigraded Krasil'shchik-Schouten bracket.

In particular, this construction would associate to a divergence operator on a supermanifold $(M, \mathcal{A})$ a generator of the bigraded bracket on multivectors on the supermanifold. We hope to return to this question and also to study the relationship between the generators of a graded bracket and those of its derived brackets, in the sense of [20], in a future publication.

\section{ApPendix. The BerEZINIAN SHEAF}

We shall recall the definition of the berezinian integral and some fundamental results, following [4], [30], [35], [41] and mostly [12] and [13].

Let $(M, \mathcal{A})$ be a supermanifold of dimension $m \mid n$, in the sense of [23]. Thus, $M$ is a smooth manifold and $\mathcal{A}$ is a sheaf of $\mathbb{Z}_{2}$-graded commutative, associative $\mathbb{R}$-algebras over $M$. There is an exact sequence

$$
0 \rightarrow \mathcal{N} \rightarrow \mathcal{A} \rightarrow \mathcal{A} / \mathcal{N} \rightarrow 0
$$

where $\mathcal{N}$ is the sheaf of nilpotent sections of $\mathcal{A}$, and $\mathcal{A} / \mathcal{N}$ is the sheaf $C^{\infty}(M)$, regarded as trivially graded. The projection $\mathcal{A} \rightarrow \mathcal{A} / \mathcal{N}=C^{\infty}(M)$ is denoted by the symbol $\sim$, and there is a unique prolongation to the module of differential forms of this projection, that commutes with the de Rham differentials. Thus, if we denote by $\mathrm{d}$ and $\mathrm{d}^{G}$ the de Rham differentials in $M$ and $(M, \mathcal{A})$, then

$$
\widetilde{\mathrm{d}^{G} \alpha}=\mathrm{d} \widetilde{\alpha},
$$

for any differential form $\alpha$ on the supermanifold $(M, \mathcal{A})$.

The berezinian sheaf can be described as follows. Let $\mathcal{P}^{k}(\mathcal{A})$ be the vector space of the differential operators of order $k$ on $\mathcal{A}$. There is both a right and a left $\mathcal{A}$ module structure on $\mathcal{P}^{k}(\mathcal{A})$ given by $(f . P)(g)=f . P(g)$ and $(P . f)(g)=P(f . g)$, respectively, for sections $f, g$ of $\mathcal{A}$ and $P \in \mathcal{P}^{k}(\mathcal{A})$. If $\left(x^{1}, \ldots, x^{m}, s^{1}, \ldots, s^{n}\right)$ are graded coordinates on an open set $U$ in $M$, then $\left.\mathcal{P}^{k}(\mathcal{A})\right|_{U}$ is free for both structures of $\mathcal{A}$-module, with basis

$$
\left(\frac{\partial}{\partial x^{1}}\right)^{k_{1}} \circ \cdots \circ\left(\frac{\partial}{\partial x^{m}}\right)^{k_{m}} \circ \frac{\partial}{\partial s^{\rho_{1}}} \circ \cdots \circ \frac{\partial}{\partial s^{\rho_{j}}},
$$

where $k_{1}, \ldots, k_{m} \in \mathbb{N}, 1 \leq \rho_{1}<\rho_{2}<\ldots<\rho_{j} \leq n$ and $k_{1}+\cdots+k_{m}+j=k$.

Let us now define $\mathcal{P}^{k}\left(\mathcal{A}, \Omega_{\mathcal{A}}^{m}\right)=\Omega_{\mathcal{A}}^{m} \otimes \mathcal{P}^{k}(\mathcal{A})$, where $\Omega_{\mathcal{A}}^{m}$ is the sheaf of differential $m$-forms on $(M, \mathcal{A})$. Let $\mathcal{K}^{n}$ be the subsheaf of elements $\mathbf{P}$ in $\mathcal{P}^{n}\left(\mathcal{A}, \Omega_{\mathcal{A}}^{m}\right)$ such that, for any section $f$ of $\mathcal{A}$ over an open set $U$ of $M$ with compact support, there exists an $(m-1)$-differential form $\omega$ with compact support in $U$ such that $\widetilde{\mathbf{P}(f)}=\mathrm{d} \omega$. Is is easy to show that $\mathcal{K}^{n}$ is a subsheaf of right $\mathcal{A}$-modules of $\mathcal{P}^{n}\left(\mathcal{A}, \Omega_{\mathcal{A}}^{m}\right)$. The berezinian sheaf is the quotient sheaf $\mathcal{P}^{n}\left(\mathcal{A}, \Omega_{\mathcal{A}}^{m}\right) / \mathcal{K}^{n}$. The sections of this sheaf can be locally expressed as

$$
\left[\mathrm{d}^{G} x^{1} \wedge \cdots \wedge \mathrm{d}^{G} x^{m} \otimes \frac{\partial}{\partial s^{1}} \circ \cdots \circ \frac{\partial}{\partial s^{n}}\right] . f,
$$


where $f$ is a section of $\mathcal{A}$. If $V \subset M$ is an open set with graded coordinates $\left(y^{1}, \ldots, y^{m}, t^{1}, \ldots, t^{n}\right)$, then, on $U \cap V$,

$\left[\mathrm{d}^{G} y^{1} \wedge \cdots \wedge \mathrm{d}^{G} y^{m} \otimes \frac{\partial}{\partial t^{1}} \circ \cdots \circ \frac{\partial}{\partial t^{n}}\right]=\left[\mathrm{d}^{G} x^{1} \wedge \cdots \wedge \mathrm{d}^{G} x^{m} \otimes \frac{\partial}{\partial s^{1}} \circ \cdots \circ \frac{\partial}{\partial s^{n}}\right] \cdot \operatorname{Ber}\left(\begin{array}{ll}A & B \\ C & D\end{array}\right)$

where

$$
\left(\begin{array}{ll}
A & B \\
C & D
\end{array}\right)=\left(\begin{array}{ll}
\left(\frac{\partial y^{i}}{\partial x^{j}}\right) & \left(\frac{\partial t^{\rho}}{\partial x^{j}}\right) \\
\left(\frac{\partial y^{i}}{\partial s^{\sigma}}\right) & \left(\frac{\partial t^{\rho}}{\partial s^{\sigma}}\right)
\end{array}\right)
$$

and where Ber denotes the berezinian. (The berezinian, or superdeterminant, of an invertible even matrix of the form $\left(\begin{array}{ll}A & B \\ C & D\end{array}\right)$ is $\operatorname{det}\left(A-B D^{-1} C\right) \operatorname{det}\left(D^{-1}\right)$.)

If $M$ is an orientable smooth manifold, the Berezin integral, $\int_{(M, \mathcal{A})}$, maps the sections with compact support of the berezinian sheaf to $\mathbb{R}$, and is defined by $\int_{(M, \mathcal{A})}[\mathbf{P}]=\int_{M} \widetilde{\mathbf{P}(1)}$. As an example, if $(M, \mathcal{A})=\mathbb{R}^{m \mid n}$, then

$\int_{\mathbb{R}^{m \mid n}}\left[\mathrm{~d}^{G} x^{1} \wedge \cdots \wedge \mathrm{d}^{G} x^{m} \otimes \frac{\partial}{\partial s^{1}} \circ \cdots \circ \frac{\partial}{\partial s^{n}}\right] . f=(-1)^{\frac{n(n-1)}{2}} \int_{\mathbb{R}^{n}} f_{(1,2, \ldots, n)}(x) \mathrm{d} x^{1} \wedge \cdots \wedge \mathrm{d} x^{m}$,

where $f_{(1,2, \ldots, n)}$ is the coefficient of $s^{1} s^{2} \ldots s^{n}$ in the expansion of $f$ as a sum of products of the $s^{\rho}$ 's.

A section, $\xi$, of the berezinian sheaf is called a berezinian volume if it is a generator of the berezinian sheaf, i.e., if any other section can be uniquely written as $\xi . f$ for some section $f$ of $\mathcal{A}$. A berezinian volume is a homogeneous section of the berezinian sheaf, whose degree depends on the parity of the dimension $n$. If $\xi$ is a berezinian volume and $v$ is a section of $\mathcal{A}$, then $\xi . v$ is also a berezinian volume if and only $v$ is invertible and even.

In order to define the Lie derivatives of berezinian volumes with respect to graded vector fields, we first observe that, in a similar way, we can define the right submodule $\mathcal{K}^{n+k}$ of $\mathcal{P}^{n+k}\left(\mathcal{A}, \Omega_{\mathcal{A}}^{m}\right)$, for each $k \geq 1$, and that the canonical inclusion $\mathcal{P}^{n}\left(\mathcal{A}, \Omega_{\mathcal{A}}^{m}\right) \hookrightarrow \mathcal{P}^{n+k}\left(\mathcal{A}, \Omega_{\mathcal{A}}^{m}\right)$ induces an isomorphism of sheaves of right $\mathcal{A}$-modules from $\mathcal{P}^{n}\left(\mathcal{A}, \Omega_{\mathcal{A}}^{m}\right) / \mathcal{K}^{n}$ to $\mathcal{P}^{n+k}\left(\mathcal{A}, \Omega_{\mathcal{A}}^{m}\right) / \mathcal{K}^{n+k}$.

Let $D$ be a graded vector fied on $(M, \mathcal{A})$. The Lie derivative of the berezinian volume $[\omega \otimes P]$ with respect to $D$ is

$$
\mathcal{L}_{D}[\omega \otimes P]=(-1)^{|D||\omega \otimes P|+1}[\omega \otimes P \circ D] .
$$

The main properties of the Lie derivatives of berezinian volumes are stated in Section 2, and are used there in order to derive the properties of the divergence operators.

Acknowledgments. Y. K.-S. would like to thank P. Cartier, J. Huebschmann, D. Leites, H. Khudaverdian, A. Schwarz, J. Stasheff and T. Voronov for interesting exchanges on the topic of this work. Thanks are also due to the referee who identified various weaknesses in the first version of this paper.

J. M. is partially supported by Pla Valencià de Ciència i Tecnologia, grant \#POST9901-30, and by DGICYT, grant \#PB97-1386. 


\section{REFERENCES}

[1] Alexandrov, M., Kontsevich, M., Schwarz, A., Zaboronsky, O., The geometry of the master equation and topological quantum field theory, Int. J. Mod. Phys. A12 (1997), 1405-1429.

[2] Batalin, I. A., Vilkovisky, G. A., Gauge algebra and quantization, Phys. Lett. B 102 (1981), $27-31$.

[3] Batalin, I. A., Vilkovisky, G. A., Closure of the gauge algebra, generalized Lie equations and Feynman rules, Nuclear Physics B 234 (1984), 106-124.

[4] Berezin, F. A., Introduction to Superanalysis, D. Reidel (1987).

[5] Beltrán, J. V., Monterde, J., Graded Poisson structures on the algebra of differential forms, Comment. Math. Helv. 70 (1995), 383-402.

[6] Beltrán, J. V., Monterde, J., Sánchez-Valenzuela, O. A., Graded Jacobi operators on the algebra of differential forms, Compositio Math. 106 (1997), 43-59.

[7] P. Deligne et al., eds., Quantum Fields and Strings: A Course for Mathematicians, Amer. Math. Soc. (1999), vol. 1, part 1.

[8] DeWitt, B., Supermanifolds, Cambridge Univ. Press (1984).

[9] Frölicher, A., Nijenhuis, A., Theory of vector-valued differential forms, part I, Indag. Math., 18 (1956), 338-359.

[10] Getzler, E., Batalin-Vilkovisky algebras and two-dimensional topological field theories, Commun. Math. Phys. 159 (1994), 265-285.

[11] Hata, H., Zwiebach, B., Developing the covariant Batalin-Vilkovisky approach to string theory, Ann. Phys. 229 (1994), 177-216.

[12] Hernández Ruipérez, D., Muñoz Masqué, J., Construction intrinsèque du faisceau de Berezin d'une variété graduée, Comptes Rendus Acad. Sci. Paris, Sér. I Math. 301 (1985), 915-918.

[13] Hernández Ruipérez, D., Muñoz Masqué, J., Variational Berezinian problems and their relationship with graded variational problems, Diff. Geometric Methods in Math. Phys. (Salamanca 1985), Lect. Notes Math. 1251, Springer-Verlag (1987), 137-149.

[14] Huebschmann, J., Poisson cohomology and quantization, J. für die reine und angew. Math. 408 (1990), 57-113.

[15] Huebschmann, J., Lie-Rinehart algebras, Gerstenhaber algebras, and Batalin-Vilkovisky algebras, Ann. Inst. Fourier 48 (1998), 425-440.

[16] Huebschmann, J., Duality for Lie-Rinehart algebras and the modular class, J. für die reine und angew. Math. 510 (1999), 103-159.

[17] Khudaverdian, O. M., Geometry of superspace with even and odd brackets, J. Math. Phys. 32 (1991), 1934-1937.

[18] Khudaverdian, O. M., Batalin-Vilkovisky formalism and odd symplectic geometry, Geometry and integrable models (Dubna 1994), Pyatov, P. N., Solodukhin, S. N., eds., World Sci. Publish. (1996), 144-181.

[19] Khudaverdian, O. M., Nersessian, A. P., On the geometry of the Batalin-Vilkovisky formalism, Mod. Phys. Lett. A 8 (1993), 2377-2385.

[20] Kosmann-Schwarzbach, Y., From Poisson algebras to Gerstenhaber algebras, Ann. Inst. Fourier 46 (1996), 1243-1274.

[21] Kosmann-Schwarzbach, Y., Modular vector fields and Batalin-Vilkovisky algebras, Banach Center Publications 51 (2000), 109-129.

[22] Kosmann-Schwarzbach, Y., Magri, F., Poisson-Nijenhuis structures, Ann. Inst. Henri Poincaré A53 (1990), 35-81.

[23] Kostant, B., Graded manifolds, graded Lie theory and prequantization, Proc. Conf. Diff. Geom. Methods in Math. Phys. (Bonn 1975), Lecture Notes Math. 570, Springer-Verlag (1977), 177-306.

[24] Koszul, J.-L., Crochet de Schouten-Nijenhuis et cohomologie, in Élie Cartan et les mathématiques d'aujourd'hui, Astérisque, hors série, Soc. Math. Fr. (1985), 257-271.

[25] Krasil'shchik, I. S., Schouten brackets and canonical algebras, Lecture Notes Math. 1334, Springer-Verlag (1988), 79-110.

[26] Krasil'shchik, I. S., Supercanonical algebras and Schouten brackets, Mat. Zametki, 49(1) (1991), 70-76, Mathematical Notes 49(1) (1991), 50-54. 
[27] Leites, D., Supermanifold Theory, Karelia Branch of the USSR Acad. of Sci., Petrozavodsk, (1983) (in Russian).

[28] Leites, D., Quantization and supermanifolds, Supplement 3 in Berezin, F. A., Shubin, M. A., The Schrödinger Equation, Kluwer (1991).

[29] Lian, B. H., Zuckerman, G. J., New perspectives on the BRST-algebraic structure of string theory, Commun. Math. Phys. 154 (1993), 613-646.

[30] Manin, Y. I., Gauge Field Theory and Complex Geometry, Springer-Verlag (1988).

[31] Manin, Y. I., Penkov, I. B., The formalism of left and right connections on supermanifolds, Lectures on Supermanifolds, Geometrical Methods and Conformal Groups, Doebner, H.-D., Hennig, J. D., Palev, T. D., eds., World Sci. Publ. (1989), 3-13.

[32] Monterde, J., Montesinos, A., Integral curves of derivations, Ann. Global Anal. Geom. 6 (1988), 177-189.

[33] Monterde, J., Sánchez Valenzuela, A., The exterior derivative as a Killing vector field, Israel J. Math. 93 (1996), 157-170.

[34] Penkov, I. B., D-modules on supermanifolds, Invent. Math. 71 (1983), 501-512.

[35] Rothstein, M., Integration on noncompact supermanifolds, Trans. Amer. Math. Soc. 299 (1987), 387-396.

[36] Schechtman, V., Remarks on formal deformations and Batalin-Vilkovisky algebras, preprint math.AG/9802006.

[37] Schwarz, A., Geometry of Batalin-Vilkovisky quantization, Commun. Math. Phys. 155 (1993), 249-260.

[38] Schwarz, A., Semi-classical approximation in Batalin-Vilkovisky formalism, Commun. Math. Phys. 158 (1993), 373-396.

[39] Stasheff, J., Deformation theory and the Batalin-Vilkovisky master equation, Deformation Theory and Symplectic Geometry (Ascona 1996), Sternheimer, D., Rawnsley, J., Gutt, S., eds., Kluwer (1997), 271-284.

[40] Vaisman, I., Lectures on the Geometry of Poisson Manifolds, Birkhäuser, 1994.

[41] Voronov, T., Geometric integration theory on supermanifolds, Sov. Sci. Rev., C Math., 9 (1992), 1-138.

[42] Weinstein, A., The modular automorphism group of a Poisson manifold, J. Geom. Phys. 23 (1997), 379-394.

[43] Witten, E., A note on the antibracket formalism, Mod. Phys. Lett. A5 (1990), 487-494.

[44] Xu, P., Gerstenhaber algebras and BV-algebras in Poisson geometry, Commun. Math. Phys. 200 (1999), 545-560.

Centre de Mathématiques (U.M.R. 7640 du C.N.R.S.), Ecole Polytechnique, F-91128

Palaiseau, France

E-mail address: yks@math.polytechnique.fr

Dpto. De Geometría y Topología, Universitat de València, E-46100 Burjasot (VALÈncia), Spain

E-mail address: monterde@vm.ci.uv.es 